\title{
Microwave-assisted one-pot synthesis of benzothiazole and benzoxazole libraries as analgesic agents
}

\author{
C PRAVEEN $^{\mathrm{a}}$, A NANDAKUMAR ${ }^{\mathrm{a}}$, P DHEENKUMAR $^{\mathrm{b}}$, D MURALIDHARAN $^{\mathrm{a}}$ and \\ P T PERUMAL ${ }^{\mathrm{a}, *}$ \\ ${ }^{a}$ Organic Chemistry Division, Central Leather Research Institute (CSIR), Adyar, Chennai 600 020, India \\ ${ }^{b}$ Department of Pharmaceutical Chemistry, EGS Pillay College of Pharmacy, Nagapattinam 611 002, India \\ e-mail: ptperumal@gmail.com
}

MS received 28 August 2011; revised 19 November 2011; accepted 2 December 2011

\begin{abstract}
Microwave-assisted synthesis of benzothiazole and benzoxazole libraries via PIFA promoted cyclocondensation of 2-aminothiophenols/2-aminophenols with aldehydes under one-pot condition in good to excellent yields was achieved. Twenty compounds have been investigated for their analgesic activity and showed moderate to good activity.
\end{abstract}

Keywords. Microwave; one-pot synthesis; benzothiazoles; benzoxazoles; analgesic activity.

\section{Introduction}

The biaryl pharmacophore of benzothiazole and benzoxazole exhibits a wide range of biological properties and has led to continued interest in medicinal chemistry. ${ }^{1}$ The benzothiazolyl moiety is a component in various antagonists like $\mathrm{Ca}^{2+}$ channel, ${ }^{2} \mathrm{LTD}_{4}{ }^{3}$ and orexin receptor. ${ }^{4}$ Benzothiazoles are known to inhibit several enzymes such as acetyl cholinesterase, ${ }^{5}$ monamine oxidase, ${ }^{6-9}$ lipoxygenase, ${ }^{10}$ lysophosphatidic acid acyltransferase- $\beta,{ }^{11}$ aldose reductase, ${ }^{12}$ cyclooxygenase, ${ }^{13}$ carbonic anhydrase, ${ }^{14} \mathrm{H}^{+}-\mathrm{K}^{+}$ATPase,${ }^{15} \mathrm{HCV}$ helicase, ${ }^{16}$ protease $^{17}$ and stearoyl-coA $\delta-9$ desaturase. ${ }^{18}$ Other recognized pharmacological activities of benzothiazoles include antitumour, ${ }^{19}$ antimicrobial, ${ }^{20}$ antioxidant ${ }^{21}$ and antiglutamate. ${ }^{22}$ On the other hand, benzoxazole moiety is found in various pharmaceuticals displaying a broad spectrum of biological activity including antiinflammatory, antitumour, antirheumatic, antimicrobial and antiviral effects. ${ }^{23}$ Other significant physiological activities associated with benzoxazoles are H37Rv inhibitory, ${ }^{24}$ elastase inhibitory, ${ }^{25}$ $5 \mathrm{HT}_{3}$ receptor agonist ${ }^{26}$ and cytotoxicity towards $\mathrm{P} 338$ cells. ${ }^{27}$

The most useful synthesis of benzothiazoles include condensation of 2-aminobenzenethiol with carboxyl derivatives, ${ }^{28}$ cyclization of ortho-haloanilides, ${ }^{29}$

*For correspondence the radical cyclization of thioacylbenzanilides, ${ }^{30}$ DMP mediated intramolecular cyclization of thioformanilides. ${ }^{31}$ Apart from these general methods, there have been many methodologies disclosed in the literature for the construction of benzothiazoles. ${ }^{32-40}$ On the other hand, the classical approach for the synthesis of benzoxazoles involves (i) coupling of carboxylic acids with 2 -aminophenols by dehydration catalysed by strong acid; ${ }^{41}$ and (ii) the oxidative cyclization of phenolic Schiff's bases derived from the condensation of 2-aminophenols and aldehydes, using various oxidants such as $\mathrm{PhI}(\mathrm{OAc})_{2},{ }^{42} \mathrm{Mn}(\mathrm{OAc})_{3},{ }^{43} \mathrm{ThClO}_{4},{ }^{44}$ $\mathrm{Ba}\left(\mathrm{MnO}_{4}\right)_{2},{ }^{45} \mathrm{NiO}_{2},{ }^{46} \mathrm{~Pb}(\mathrm{OAc})_{4},{ }^{47} \mathrm{DDQ},{ }^{48} \mathrm{NIS},{ }^{49}$ $\mathrm{Cu}-\mathrm{np},{ }^{50}$ aerial oxidation in the presence of activated carbon (Darco KB), ${ }^{51} \mathrm{CuCl}^{52}$ and $\mathrm{Pd} / \mathrm{Pt}-\mathrm{C} .{ }^{53}$ Other synthetic routes for benzoxazole include microwaveassisted reaction of $\mathrm{N}$-acyl-aminophenol, ${ }^{54}$ iron catalysed intramolecular $O$-arylation of 2-haloacetanilides ${ }^{55}$ and cyclization of phenolic Schiff base under UV irradiation. ${ }^{56}$ Apart from these methodologies, there have been many precedents for the synthesis of 2-aryl benzothiazoles and benzoxazoles via palladium catalysed cross-coupling reactions have been disclosed in the literature. ${ }^{57}$

The synthetic scope of the aforesaid protocols is limited by two factors: (i) lack of general procedure that supports the synthesis of both benzothiazoles and benzoxazoles, (ii) lack of practical synthesis supporting structurally diverse spacers and substitution patterns in the target library. Thus, it is clear that an efficient protocol is needed. In the context of heterocycle 
synthesis, ${ }^{58}$ we have already addressed this problem by employing PCC as an effective oxidant for the synthesis of both benzothiazoles and benzoxazoles via oxidative cyclization of thiophenolic and phenolic Schiff's bases, respectively. ${ }^{58 a}$ Although the substrate scope was modestly explored, this procedure is limited by the powerful oxidizing ability of PCC and the potential carcinogenicity of $\mathrm{Cr}^{6+}$. In order to circumvent this limitation, we envisaged an alternate oxidant for the synthesis of benz(oxa)thiazoles. In this paper, we report PIFA [phenyliodonium bis(trifluoroacetate)] as an effective oxidant for the one-pot synthesis of benz(oxa)thiazoles via cyclocondensation of 2-aminothiophenols/2-aminophenols with aldehydes under microwave irradiation. Representative compounds were screened for their in vivo analgesic activity and the results were presented.

\section{Experimental}

\subsection{Materials, methods and instruments}

Melting points were determined on Gallenkamp melting point apparatus and are uncorrected. Infrared (IR) spectra were recorded on a Perkin-Elmer FTIR spectrophotometer as $\mathrm{KBr}$ pellets. ${ }^{1} \mathrm{H}$ and ${ }^{13} \mathrm{C}$ NMR spectra were obtained in $\mathrm{CDCl}_{3}$ and DMSO- $d_{6}$ on a JEOL spectrometer at 500 and $125 \mathrm{MHz}$, respectively. Proton chemical shifts $(\delta)$ are relative to tetramethylsilane (TMS, $\delta=0.00$ ) as internal standard and expressed in parts per million. The number of protons $(n)$ for a given resonance was indicated as $n \mathrm{H}$. Coupling constants $(J)$ are given in hertz. Spin multiplicities are given as $s$ (singlet), $d$ (doublet), $t$ (triplet) and $m$ (multiplet). Mass spectra were recorded on a Thermo Finnigan LCQ Advantage MAX 6000 ESI mass spectrometer and Perkin-Elmer GC-MS. Elemental analyses were recorded using a Thermo Finnigan FLASH EA $1112 \mathrm{CHN}$ analyzer. All the compounds gave $\mathrm{C}, \mathrm{H}$ and $\mathrm{N}$ analysis within $\pm 0.5 \%$ of the theoretical values. All microwave experiments were performed using an Emrys Optimizer in 2-5 mL pyrex reaction vessels. Each vessel contained a Teflon stir bar and Tefloncoated reaction vessel cap. Column chromatography was performed using a mixture of petroleum ether and ethyl acetate on silica gel (100-200 mesh, SRL, India). Analytical TLC was performed on precoated plastic sheets of silica gel G/UV-254 of $0.2 \mathrm{~mm}$ thickness (Macherey-Nagel, Germany) using analytical grade solvents and visualizing with iodine spray $\left(10 \%(\mathrm{w} / \mathrm{w}) \mathrm{I}_{2}\right.$ in silica gel) or UV light ( $\lambda=254$ and $365 \mathrm{~nm})$.

\subsection{General procedure for the synthesis of benzothiazoles $2 a-2 z$}

To a pyrex reaction vessel were added 2aminothiophenol (1.1 mmol), aldehyde $(1.0 \mathrm{mmol})$ and PIFA $(1.05 \mathrm{mmol})$ in ethanol $(3 \mathrm{ml})$. The reaction vessel was then placed in the Emrys Optimizer and exposed to microwave irradiation $\left(80^{\circ} \mathrm{C}\right)$ for $15 \mathrm{~min}$. The reaction mixture was then allowed to cool at room temperature and quenched with $15 \mathrm{~mL}$ of water. The crude reaction mixture was extracted with EtOAc (3 $\times 15 \mathrm{~mL}$ ). The combined organic layers were dried over anhydrous $\mathrm{Na}_{2} \mathrm{SO}_{4}$, filtered, concentrated and purified by column chromatography on silica gel using petroleum ether/EtOAc to afford the pure product.

2.2a 2-(4-Methoxyphenyl)benzo[d]thiazole (2a): Yellow solid; mp $122-124^{\circ} \mathrm{C} ; R_{f}=0.59$ (AcOEt/ petroleum ether 10\%). IR (KBr): 3023, 2996, 2900, 2836, 1605, 1521, 1485, 1260, $832 \mathrm{~cm}^{-1}$. ${ }^{1} \mathrm{H}$ NMR $\left(500 \mathrm{MHz}, \mathrm{CDCl}_{3}\right) \delta_{\mathrm{H}} 3.91\left(\mathrm{~s}, 3 \mathrm{H},-\mathrm{OCH}_{3}\right) ; 7.00-7.05$ $(\mathrm{m}, 2 \mathrm{H}, \mathrm{Ar}-\mathrm{H}) ; 7.35(\mathrm{~d}, 1 \mathrm{H}, J=7.6 \mathrm{~Hz}, \mathrm{Ar}-\mathrm{H}) ; 7.50$ (d, $1 \mathrm{H}, J=7.6 \mathrm{~Hz}, \mathrm{Ar}-\mathrm{H})$; 7.91-8.04 (m, 4H, Ar-H). ${ }^{13} \mathrm{C}$ NMR $\left(125 \mathrm{MHz}, \mathrm{CDCl}_{3}\right) \delta_{\mathrm{C}} 55.5,114.4,121.6$, $122.9,124.9,126.3,126.5,129.2,135.0,154.3,162.0$, 167.9. MS (EI): $m / z=241\left[\mathrm{M}^{+}\right]$. Anal. Calcd for $\mathrm{C}_{14} \mathrm{H}_{11}$ NOS: C, 69.68; H, 4.59; N, 5.80\%. Found: C, $69.89 ; \mathrm{H}, 4.54 ; \mathrm{N}, 5.72 \%$.

\section{2b 2-(4-(Trifluoromethyl)phenyl)benzo[d]thiazole}

(2b): Colourless solid; mp $158-160^{\circ} \mathrm{C} ; R_{f}=0.35$ (AcOEt/petroleum ether 40\%). IR (KBr): 3058, 2898, $1658,1154,1071,798 \mathrm{~cm}^{-1}$. ${ }^{1} \mathrm{H}$ NMR $(500 \mathrm{MHz}$, $\left.\mathrm{CDCl}_{3}\right) \delta_{\mathrm{H}} 7.40-7.45(\mathrm{~m}, 1 \mathrm{H}, \mathrm{Ar}-\mathrm{H}) ; 7.50-7.54(\mathrm{~m}$, $1 \mathrm{H}, \mathrm{Ar}-\mathrm{H}) ; 7.77$ (d, 2H, $J=8.4 \mathrm{~Hz}, \mathrm{Ar}-\mathrm{H}) ; 7.93$ (ddd, $1 \mathrm{H}, J=0.8,0.8,8.4 \mathrm{~Hz}, \mathrm{Ar}-\mathrm{H}) ; 8.11$ (ddd, $1 \mathrm{H}, J=$ $1.1,1.1,7.1 \mathrm{~Hz}, \mathrm{Ar}-\mathrm{H}) ; 8.20(\mathrm{dd}, 2 \mathrm{H}, J=0.7,8.8 \mathrm{~Hz}$, Ar-H). ${ }^{13} \mathrm{C}$ NMR $\left(125 \mathrm{MHz}, \mathrm{CDCl}_{3}\right) \delta_{\mathrm{C}} 121.8,123.4$, $124.5\left(J_{\mathrm{C}-\mathrm{F}}=268.0 \mathrm{~Hz}\right), 125.5,126.0\left(J_{\mathrm{C}-\mathrm{F}}=3.6 \mathrm{~Hz}\right)$, $126.7,132.4\left(J_{\mathrm{C}-\mathrm{F}}=32.9 \mathrm{~Hz}\right), 135.0,136.7,154.2$, 159.9. MS (EI): $m / z=280[\mathrm{M}+\mathrm{H}]^{+}$. Anal. Calcd for $\mathrm{C}_{14} \mathrm{H}_{8} \mathrm{~F}_{3} \mathrm{NS}$ : C, 60.21; H, 2.89; N, 5.02\%. Found: C, $59.99 ; \mathrm{H}, 2.94 ; \mathrm{N}, 5.11 \%$.

\section{2c 4-(Benzo[d]thiazol-2-yl)-N,N-dimethylbvenzenamine}

(2c): Brown solid; mp $175-177^{\circ} \mathrm{C} ; \quad R_{f}=0.30$ (AcOEt/petroleum ether 20\%). IR (KBr): 3398, 1598, 1478, 1201, 1012, $981 \mathrm{~cm}^{-1}$. ${ }^{1} \mathrm{H}$ NMR $(500 \mathrm{MHz}$, $\left.\mathrm{CDCl}_{3}\right) \delta_{\mathrm{H}} 3.07\left(\mathrm{~s}, 6 \mathrm{H},-\mathrm{N}\left(\mathrm{CH}_{3}\right)_{2}\right) ; 6.75(\mathrm{dd}, 2 \mathrm{H}, J=$ 7.2, $1.8 \mathrm{~Hz}, \mathrm{Ar}-\mathrm{H}) ; 7.31-7.35$ (m, 1H, Ar-H); 7.43$7.46(\mathrm{~m}, 1 \mathrm{H}$, Ar-H); 7.85-7.88 (m, 1H, Ar-H); 8.01 (dd, $3 \mathrm{H}, J=7.2,1.8 \mathrm{~Hz}, \mathrm{Ar}-\mathrm{H}) .{ }^{13} \mathrm{C}$ NMR $(125 \mathrm{MHz}$, 
$\left.\mathrm{CDCl}_{3}\right) \delta_{\mathrm{C}} 39.9,111.5,121.2,122.3,124.0,126.0$, 128.9, 134.6, 152.0, 154.5, 168.9. MS (EI): $m / z=$ $255[\mathrm{M}+\mathrm{H}]^{+}$. Anal. Calcd for $\mathrm{C}_{15} \mathrm{H}_{14} \mathrm{~N}_{2} \mathrm{~S}: \mathrm{C}, 70.83 ; \mathrm{H}$, $5.55 ; \mathrm{N}, 11.01 \%$. Found: C, 71.01; H, 5.51; N, 10.92\%.

$2.2 \mathrm{~d} \quad$ 2-(4-Chlorophenyl)benzo[d]thiazole (2d): Yellow solid; $\mathrm{mp} 114-116^{\circ} \mathrm{C} ; R_{f}=0.50$ (AcOEt/ petroleum ether 10\%). IR (KBr): 3055, 2360, 1560, $1455,1430,1317,1275,1060,966,750,725 \mathrm{~cm}^{-1} .{ }^{1} \mathrm{H}$ NMR $\left(500 \mathrm{MHz}, \mathrm{CDCl}_{3}\right) \delta_{\mathrm{H}}$ 7.39-7.43 (m, 2H, Ar-H); 7.45-7.52 (m, 2H, Ar-H); 7.85-8.01 (m, 4H, Ar-H). ${ }^{13} \mathrm{C}$ NMR $\left(125 \mathrm{MHz}, \mathrm{CDCl}_{3}\right) \delta_{\mathrm{C}} 121.9,123.6,125.5$, 126.8, 129.0, 129.6, 132.4, 135.4, 137.1, 154.4, 166.9. MS (EI): $m / z=245\left[\mathrm{M}^{+}\right], 247\left[\mathrm{M}^{+2}\right]$. Anal. Calcd for $\mathrm{C}_{13} \mathrm{H}_{8} \mathrm{ClNS}$ : C, 63.54; H, 3.28; $\mathrm{N}, 5.70 \%$. Found: $\mathrm{C}$, $63.75 ; \mathrm{H}, 3.24 ; \mathrm{N}, 5.63 \%$.

2.2e 2-(Naphthalen-2-yl)benzo[d]thiazole (2e): Brown solid; mp 123-125 ${ }^{\circ} \mathrm{C} ; R_{f}=0.64$ (AcOEt/petroleum ether 20\%). IR (KBr): 3049, 2920, 2855, 1597, 1499, 1452, 1430, 1362, 1306, 1270, 1174, 1123, 982, 937, $880 \mathrm{~cm}^{-1} .{ }^{1} \mathrm{H}$ NMR $\left(500 \mathrm{MHz}, \mathrm{CDCl}_{3}\right) \delta_{\mathrm{H}} 7.30-7.37$ (m, 1H, Ar-H); 7.44-7.50 (m, 3H, Ar-H); 7.80-7.91 (m, $4 \mathrm{H}, \mathrm{Ar}-\mathrm{H}) ; 8.09$ (d, $1 \mathrm{H}, J=8.0 \mathrm{~Hz}, \mathrm{Ar}-\mathrm{H}) ; 8.15(\mathrm{~d}, 1 \mathrm{H}$, $J=8.4 \mathrm{~Hz}) ; 8.50(\mathrm{~s}, 1 \mathrm{H}, \mathrm{Ar}-\mathrm{H}) .{ }^{13} \mathrm{C}$ NMR $(125 \mathrm{MHz}$, $\left.\mathrm{CDCl}_{3}\right) \delta_{\mathrm{C}} 121.6,123.2,124.4,125.2,126.7,127.4$, 127.5, 127.9, 128.8, 130.9, 133.1, 134.6, 135.1, 154.2, 168.1. MS (EI): $m / z=261\left[\mathrm{M}^{+}\right]$. Anal. Calcd for $\mathrm{C}_{17} \mathrm{H}_{11} \mathrm{NS}$ : C, 78.13; H, 4.24; N, 5.36\%. Found: C, 78.30; H, 4.17; N, 5.29\%.

2.2f Methyl 4-(benzo[d]thiazol-2-yl)benzoate (2f): Colourless solid; mp $148-151^{\circ} \mathrm{C} ; \quad R_{f}=0.25$ (AcOEt/petroleum ether 30\%). IR (KBr): 3360, 3270, 2933, 2853, 1724, 1679, 751, $724 \mathrm{~cm}^{-1} ;{ }^{1} \mathrm{H}$ NMR $\left(500 \mathrm{MHz}, \mathrm{CDCl}_{3}\right) \delta_{\mathrm{H}} 3.99\left(\mathrm{~s}, 3 \mathrm{H},-\mathrm{COOCH}_{3}\right) ; 6.94-$ $6.99(\mathrm{td}, 1 \mathrm{H}, J=7.6,1.3 \mathrm{~Hz}, \mathrm{Ar}-\mathrm{H}) ; 7.26-7.29(\mathrm{~m}$, $1 \mathrm{H}, \mathrm{Ar}-\mathrm{H}) ; 7.45-7.49$ (dd, $1 \mathrm{H}, J=7.6,1.3 \mathrm{~Hz}, \mathrm{Ar}-\mathrm{H})$; $7.70-7.73(\mathrm{~d}, 2 \mathrm{H}, J=8.3 \mathrm{~Hz}, \mathrm{Ar}-\mathrm{H}) ; 8.09-8.12(\mathrm{~d}, 2 \mathrm{H}$, $J=8.3 \mathrm{~Hz}, \mathrm{Ar}-\mathrm{H}) ; 8.42-8.46(\mathrm{~d}, 1 \mathrm{H}, J=8.3 \mathrm{~Hz}, 1 \mathrm{H})$. ${ }^{13} \mathrm{C}$ NMR $\left(125 \mathrm{MHz}, \mathrm{CDCl}_{3}\right) \delta_{\mathrm{C}} 52.5,120.7,124.0$, 124.7, 127.1, 130.0, 132.4, 133.2, 136.7, 138.0, 139.7, 164.0, 166.2. MS (EI): $m / z=270[\mathrm{M}+\mathrm{H}]^{+}$. Anal. Calcd for $\mathrm{C}_{15} \mathrm{H}_{11} \mathrm{NO}_{2} \mathrm{~S}$ : C, 66.89; $\mathrm{H}, 4.12 ; \mathrm{N}, 5.20 \%$. Found: C, 67.10; H, 4.06; N, 5.11\%.

\section{2g 5-Methoxy-2-phenylbenzo[d]thiazole (2g):} Colourless solid; mp $74-76^{\circ} \mathrm{C} ; R_{f}=0.62$ (AcOEt/ petroleum ether 15\%). IR (KBr): 2955, 2940, 2840, 1597, 1462, 1429, 1256, 1167, 1150, $1077 \mathrm{~cm}^{-1} .{ }^{1} \mathrm{H}$ NMR $\left(500 \mathrm{MHz}, \mathrm{CDCl}_{3}\right) \delta_{\mathrm{H}} 3.86\left(\mathrm{~s}, 3 \mathrm{H},-\mathrm{OCH}_{3}\right) ; 7.05$ $\left(\mathrm{dd}, 1 \mathrm{H}, J_{1}=2.6 \mathrm{~Hz}, J_{2}=9.1 \mathrm{~Hz}, \mathrm{Ar}-\mathrm{H}\right) ; 7.39-7.47(\mathrm{~m}$, $3 \mathrm{H}, \mathrm{Ar}-\mathrm{H}) ; 7.56(\mathrm{~d}, 1 \mathrm{H}, J=2.6 \mathrm{~Hz}, \mathrm{Ar}-\mathrm{H}) ; 7.70(\mathrm{~d}, 1 \mathrm{H}$,
$J=9.1 \mathrm{~Hz}, \mathrm{Ar}-\mathrm{H}) ; 8.01-8.10(\mathrm{~m}, 2 \mathrm{H}, \mathrm{Ar}-\mathrm{H}) .{ }^{13} \mathrm{C} \mathrm{NMR}$ $\left(125 \mathrm{MHz}, \mathrm{CDCl}_{3}\right) \delta_{\mathrm{C}} 55.9,106.0,115.4,122.1,127.3$, $127.7,129.3,131.1,134.1,156.0,159.5,169.4$. MS (EI): $m / z=241\left[\mathrm{M}^{+}\right]$. Anal. Calcd for $\mathrm{C}_{14} \mathrm{H}_{11}$ NOS: $\mathrm{C}$, $69.68 ; \mathrm{H}, 4.59 ; \mathrm{N}, 5.80 \%$. Found: C, 69.48; H, 4.61; N, $5.87 \%$.

2.2h 2-(3-Nitrophenyl)benzo[d]thiazole (2h): Colourless solid; mp $181-183^{\circ} \mathrm{C} ; \quad R_{f}=0.50$ (AcOEt/petroleum ether 30\%). IR (KBr): 3402, 2937, 1529, 1461, 1347, 1107, 1048, $731 \mathrm{~cm}^{-1}$. ${ }^{1} \mathrm{H}$ NMR $\left(500 \mathrm{MHz}, \mathrm{CDCl}_{3}\right) \delta_{\mathrm{H}} 7.42(\mathrm{t}, 1 \mathrm{H}, J=7.6 \mathrm{~Hz}, \mathrm{Ar}-\mathrm{H})$; $7.51(\mathrm{t}, 1 \mathrm{H}, J=7.6 \mathrm{~Hz}, \mathrm{Ar}-\mathrm{H}) ; 7.65(\mathrm{t}, 1 \mathrm{H}, J=7.6 \mathrm{~Hz}$, Ar-H); 7.92 (d, 1H, $J=7.6 \mathrm{~Hz}, \mathrm{Ar}-\mathrm{H}) ; 8.09$ (d, 1H, $J$ $=7.6 \mathrm{~Hz}, \mathrm{Ar}-\mathrm{H}) ; 8.30$ (dd, $1 \mathrm{H}, J=6.9,9.2 \mathrm{~Hz}, \mathrm{Ar}-\mathrm{H})$; $8.38(\mathrm{~d}, 1 \mathrm{H}, J=7.6 \mathrm{~Hz}, \mathrm{Ar}-\mathrm{H}) ; 8.90$ (s, $1 \mathrm{H}, \mathrm{Ar}-\mathrm{H})$. ${ }^{13} \mathrm{C}$ NMR $\left(125 \mathrm{MHz}, \mathrm{CDCl}_{3}\right) \delta_{\mathrm{C}} 121.9,122.4,123.8$, 125.2, 126.1, 126.9, 130.2, 133.1, 135.3, 135.4, 148.8, 154.0, 164.9. MS (EI): $m / z=256\left[\mathrm{M}^{+}\right]$. Anal. Calcd for $\mathrm{C}_{13} \mathrm{H}_{8} \mathrm{~N}_{2} \mathrm{SO}_{2}: \mathrm{C}, 60.92 ; \mathrm{H}, 3.15 ; \mathrm{N}, 10.93 \%$. Found: C, $60.75 ; \mathrm{H}, 3.22 ; \mathrm{N}, 10.89 \%$.

\section{2i 2-(2-Methoxyphenyl)benzo[d]thiazole (2i):}

Colourless solid; mp $123-125^{\circ} \mathrm{C} ; \quad R_{f}=0.55$ (AcOEt/petroleum ether 10\%). IR (KBr): 3024, 2999, 2900, 2837, 1604, 1521, 1485, 1260, $831 \mathrm{~cm}^{-1} .{ }^{1} \mathrm{H}$ NMR $\left(500 \mathrm{MHz}, \mathrm{CDCl}_{3}\right) \delta_{\mathrm{H}} 3.99\left(\mathrm{~s}, 3 \mathrm{H},-\mathrm{OCH}_{3}\right)$; 7.10-7.55 (m, 4H, Ar-H); 7.92-8.49 (m, 4H, Ar-H). ${ }^{13} \mathrm{C}$ NMR $\left(125 \mathrm{MHz}, \mathrm{CDCl}_{3}\right) \delta_{\mathrm{C}} 55.5,111.5,120.9$, 121.0, 122.6, 124.4, 125.7, 129.3, 131.5, 135.9, 152.0, 157.0, 162.9. MS (EI): $m / z=242[\mathrm{M}+\mathrm{H}]^{+}$. Anal. Calcd for $\mathrm{C}_{14} \mathrm{H}_{11} \mathrm{NOS}$ : C, 69.68; H, 4.59; N, $5.80 \%$. Found: C, 69.91; H, 4.53; N, 5.70\%.

2.2j 2-(2-Chlorophenyl)benzo[d]thiazole (2j): Colourless solid; mp $71-73^{\circ} \mathrm{C} ; \quad R_{f}=0.39$ (AcOEt/petroleum ether 10\%). IR (KBr): 3053, 2359, 1559, 1454, 1429, 1316, 1270, 1059, 965, 749, $726 \mathrm{~cm}^{-1} .{ }^{1} \mathrm{H}$ NMR $\left(500 \mathrm{MHz}, \mathrm{CDCl}_{3}\right) \delta_{\mathrm{H}} 7.38-7.44$ $(\mathrm{m}, 3 \mathrm{H}, \mathrm{Ar}-\mathrm{H}) ; 7.51-7.54(\mathrm{~m}, 2 \mathrm{H}, \mathrm{Ar}-\mathrm{H}) ; 7.93(\mathrm{~d}$, $1 \mathrm{H}, J=7.6 \mathrm{~Hz}, \mathrm{Ar}-\mathrm{H}) ; 8.13(\mathrm{~d}, 1 \mathrm{H}, J=8.4 \mathrm{~Hz}, \mathrm{Ar}-$ $\mathrm{H}) ; 8.20-8.21\left(\mathrm{~m}, 1 \mathrm{H}\right.$, Ar-H). ${ }^{13} \mathrm{C}$ NMR $(125 \mathrm{MHz}$, $\left.\mathrm{CDCl}_{3}\right) \delta_{\mathrm{C}} 121.5,123.6,125.6,126.4,127.2,130.9$, $131.3,131.9,132.4,132.8,136.2,152.6,164.3$. MS (EI): $m / z=245\left[\mathbf{M}^{+}\right], 247\left[\mathbf{M}^{+2}\right]$. Anal. Calcd for $\mathrm{C}_{13} \mathrm{H}_{8}$ ClNS: $\mathrm{C}, 63.54 ; \mathrm{H}, 3.28$; N, 5.70\%. Found: C, 63.44; H, 3.33; N, 5.67\%.

$2.2 \mathrm{k} \quad$ 2-(3,4-Dimethoxyphenyl)benzo[d]thiazole $(\mathbf{2 k})$ : Colourless solid; $\mathrm{mp} 133-135^{\circ} \mathrm{C} ; \quad R_{f}=0.54$ (AcOEt/petroleum ether 20\%). IR (KBr): 2955, 2940, 2840, 1600, 1521, 1483, 1431, 1336, 1312, 1260, 1167 , $1145,1075 \mathrm{~cm}^{-1} \cdot{ }^{1} \mathrm{H}$ NMR $\left(500 \mathrm{MHz}, \mathrm{CDCl}_{3}\right) \delta_{\mathrm{H}} 3.96$ 
$\left(\mathrm{s}, 3 \mathrm{H},-\mathrm{OCH}_{3}\right) ; 4.03\left(\mathrm{~s}, 3 \mathrm{H},-\mathrm{OCH}_{3}\right) ; 6.97(\mathrm{~d}, 1 \mathrm{H}, J=$ $8.4 \mathrm{~Hz}, \mathrm{Ar}-\mathrm{H}) ; 7.35$ (t, $1 \mathrm{H}, J=7.6 \mathrm{~Hz}, \mathrm{Ar}-\mathrm{H}) ; 7.49$ (t, $1 \mathrm{H}, J=7.4 \mathrm{~Hz}, \mathrm{Ar}-\mathrm{H}) ; 7.65(\mathrm{~d}, 1 \mathrm{H} J=8.4 \mathrm{~Hz}$, Ar-H); 7.76 (s, $1 \mathrm{H}$, Ar-H); 7.89 (d, $1 \mathrm{H} J=7.6 \mathrm{~Hz}$, Ar-H); $8.00(\mathrm{~d}, 1 \mathrm{H}, J=8.0 \mathrm{~Hz}, \mathrm{Ar}-\mathrm{H}) .{ }^{13} \mathrm{C}$ NMR $\left(125 \mathrm{MHz}, \mathrm{CDCl}_{3}\right) \delta_{\mathrm{C}} 55.9,109.7,111.0,121.1,121.5$, 122.8, 124.9, 126.2, 126.6, 134.9, 149.2, 151.5, 154.0, 168.1. MS (EI): $m / z=271\left[\mathrm{M}^{+}\right]$. Anal. Calcd for $\mathrm{C}_{15} \mathrm{H}_{13} \mathrm{NO}_{2} \mathrm{~S}$ : C, 66.40; H, 4.83; N, 5.16\%. Found: C, $66.61 ; \mathrm{H}, 4.79 ; \mathrm{N}, 5.04 \%$.

2.21 2-[4-(Benzyloxy)-3-methoxyphenyl]benzo[d] thiazole (2l): Colourless solid; $\mathrm{mp} 97-99^{\circ} \mathrm{C} ; R_{f}=$ 0.63 (AcOEt/petroleum ether 30\%). IR (KBr): 3468, 2937, 1630, 1264, 1141, $997 \mathrm{~cm}^{-1}$. ${ }^{1} \mathrm{H}$ NMR $\left(500 \mathrm{MHz}, \mathrm{CDCl}_{3}\right) \delta_{\mathrm{H}} 3.94\left(\mathrm{~s}, 3 \mathrm{H},-\mathrm{OCH}_{3}\right) ; 5.22(\mathrm{~s}$, $\left.2 \mathrm{H},-\mathrm{OCH}_{2} \mathrm{C}_{6} \mathrm{H}_{5}\right) ; 6.93(\mathrm{~d}, 1 \mathrm{H}, J=8.4 \mathrm{~Hz}, \mathrm{Ar}-\mathrm{H})$; 7.31-7.39 (m, 4H, Ar-H); 7.42-7.48 (m, 3H, Ar-H); $7.51(\mathrm{dd}, 1 \mathrm{H}, J=2.3,8.4 \mathrm{~Hz}, \mathrm{Ar}-\mathrm{H}) ; 7.72(\mathrm{~d}, 1 \mathrm{H}, J=$ $2.3 \mathrm{~Hz}, \mathrm{Ar}-\mathrm{H}) ; 7.85$ (d, $1 \mathrm{H}, J=7.6 \mathrm{~Hz}, \mathrm{Ar}-\mathrm{H}) ; 8.01(\mathrm{~d}$, $1 \mathrm{H}, J=8.4 \mathrm{~Hz}, \mathrm{Ar}-\mathrm{H}) .{ }^{13} \mathrm{C} \mathrm{NMR}\left(125 \mathrm{MHz}, \mathrm{CDCl}_{3}\right)$ $\delta_{\mathrm{C}} 56.3,71.0,110.3,113.5,121.1,121.6,122.9,124.9$, 126.3, 127.1, 127.3, 128.1, 128.8, 134.9, 136.6, 149.9, 150.7, 154.2, 168.1. MS (EI): $m / z=349\left[\mathrm{M}^{+}\right]$. Anal. Calcd for $\mathrm{C}_{20} \mathrm{H}_{17} \mathrm{NSO}$ : C, 72.60; H, 4.93; N, $4.03 \%$. Found: C, 72.49; H, 4.82; N, 3.99\%.

$2.2 \mathrm{~m}$ 4-(Benzo[d]thiazol-2-yl)-2,6-methoxyphenol (2m): Colourless solid; mp $140-142^{\circ} \mathrm{C} ; R_{f}=0.59$ (AcOEt/ petroleum ether 25\%). IR (KBr): 3480, 2939, 1615, $1530,1480,1450,1427,1366,1334,1284,1211$, $1200 \mathrm{~cm}^{-1} \cdot{ }^{1} \mathrm{H}$ NMR $\left(500 \mathrm{MHz}, \mathrm{CDCl}_{3}\right) \delta_{\mathrm{H}} 3.99(\mathrm{~s}$, $\left.6 \mathrm{H},-\mathrm{OCH}_{3}\right) ; 5.98(\mathrm{~s}, 1 \mathrm{H},-\mathrm{OH}) ; 7.33-7.39(\mathrm{~m}, 3 \mathrm{H}$, Ar-H); 7.50 (t, $1 \mathrm{H} J=7.6 \mathrm{~Hz}, \mathrm{Ar}-\mathrm{H}) ; 7.89$ (d, 1H, $J$ $=7.6 \mathrm{~Hz}, \mathrm{Ar}-\mathrm{H}) ; 8.03(\mathrm{~d}, 1 \mathrm{H}, J=8.1 \mathrm{~Hz}, \mathrm{Ar}-\mathrm{H}) .{ }^{13} \mathrm{C}$ NMR $\left(125 \mathrm{MHz}, \mathrm{CDCl}_{3}\right) \delta_{\mathrm{C}} 56.6,106.6,121.5$ (2C), 122.8, 124.9, 125.1, 126.2, 134.8, 137.7, 147.3, 154.0, 168.1. MS (EI): $m / z=287\left[\mathrm{M}^{+}\right]$. Anal. Calcd for $\mathrm{C}_{15} \mathrm{H}_{13} \mathrm{NO}_{3} \mathrm{~S}: \mathrm{C}, 62.70 ; \mathrm{H}, 4.56 ; \mathrm{N}, 4.87 \%$. Found: $\mathrm{C}$, $62.88 ; \mathrm{H}, 4.51 ; \mathrm{N}, 4.81 \%$.

2.2n 2-[4-(Benzyloxy)-3,5-dimethoxyphenyl]benzo [d] thiazole (2n): Brown solid; mp $77-79^{\circ} \mathrm{C} ; R_{f}=$ 0.58 (AcOEt/petroleum ether 30\%). IR ( $\mathrm{KBr}$ ): 3432, 2915, 2369, 1623, 1590, 1406, 1329, 1240, 1118, $1019 \mathrm{~cm}^{-1}$. ${ }^{1} \mathrm{H}$ NMR $\left(500 \mathrm{MHz}, \mathrm{CDCl}_{3}\right) \delta_{\mathrm{H}} 3.93(\mathrm{~s}$, $\left.6 \mathrm{H},-\mathrm{OCH}_{3}\right) ; 5.09\left(\mathrm{~s}, 2 \mathrm{H},-\mathrm{OCH}_{2} \mathrm{C}_{6} \mathrm{H}_{5}\right) ; 7.29-7.38(\mathrm{~m}$, $6 \mathrm{H}, \mathrm{Ar}-\mathrm{H}) ; 7.46-7.50$ (m, 3H, Ar-H); 7.86 (d, 1H, $J=$ $7.6 \mathrm{~Hz}, \mathrm{Ar}-\mathrm{H}) ; 8.04(\mathrm{~d}, 1 \mathrm{H}, J=8.4 \mathrm{~Hz}, \mathrm{Ar}-\mathrm{H}) .{ }^{13} \mathrm{C}$ NMR $\left(125 \mathrm{MHz}, \mathrm{CDCl}_{3}\right) \delta_{\mathrm{C}} 56.2,76.9,104.9,121.7$, 123.1, 125.2, 126.4, 128.1, 128.3, 128.6, 129.3, 135.1,
137.6, 139.5. MS (EI): $m / z=377\left[\mathrm{M}^{+}\right]$. Anal. Calcd for $\mathrm{C}_{22} \mathrm{H}_{19} \mathrm{NSO}_{3}: \mathrm{C}, 70.00 ; \mathrm{H}, 5.07 ; \mathrm{N}, 3.71 \%$. Found: C, 69.89; H, 4.99; N, 3.82\%.

2.2o 4-(1,3-Benzo[d]thiazol-2-yl)-2-bromo-6-methoxyphenol (2o): Colourless solid; $\mathrm{mp} 184-186^{\circ} \mathrm{C} ; R_{f}$ $=0.46(\mathrm{AcOEt} /$ petroleum ether 30\%). IR (KBr): 3447 , 2922, 1510, 1416, 1292, 1183, 1022, 831, $722 \mathrm{~cm}^{-1} .{ }^{1} \mathrm{H}$ NMR $\left(500 \mathrm{MHz}, \mathrm{DMSO}-d_{6}\right) \delta_{\mathrm{H}} 3.93\left(\mathrm{~s}, 3 \mathrm{H},-\mathrm{OCH}_{3}\right)$; $7.39(\mathrm{~d}, 1 \mathrm{H}, J=7.6 \mathrm{~Hz}, \operatorname{Ar}-\mathrm{H}) ; 7.49-7.50(\mathrm{~m}, 1 \mathrm{H}$, Ar-H); 7.57 (s, 1H, Ar-H); 7.72 (s, 1H, Ar-H); 7.97$8.07(\mathrm{~m}, 2 \mathrm{H}, \mathrm{Ar}-\mathrm{H}) ; 10.32(\mathrm{~s}, 1 \mathrm{H},-\mathrm{OH}) .{ }^{13} \mathrm{C}$ NMR $\left(125 \mathrm{MHz}, \mathrm{DMSO}-d_{6}\right) \delta_{\mathrm{C}} 56.9,109.7,110.2,122.8$, 123.1, 124.1, 125.5, 125.8, 127.2, 134.9, 147.4, 149.2, 153.9, 166.5. MS (EI): $m / z=335\left[\mathrm{M}^{+}\right], 337\left[\mathrm{M}^{+2}\right]$. Anal. Calcd for $\mathrm{C}_{14} \mathrm{H}_{10} \mathrm{BrNO}_{2} \mathrm{~S}: \mathrm{C}, 50.01 ; \mathrm{H}, 3.00 ; \mathrm{N}$, 4.17\%. Found: C, 49.89; H, 3.09; N, $4.10 \%$.

\section{$2.2 \mathrm{p} \quad 2-($ Benzo $[d][1,3]$ dioxol-5-yl)benzo[d]thiazole}

(2p): Yellow solid; mp $128-130^{\circ} \mathrm{C} ; \quad R_{f}=0.60$ (AcOEt/petroleum ether 15\%). IR (KBr): 1602, 1492, 1454, 1377, 1305, 1274, 1149, 744, $699 \mathrm{~cm}^{-1}$. ${ }^{1} \mathrm{H}$ NMR $\left(500 \mathrm{MHz}, \mathrm{CDCl}_{3}\right) \delta_{\mathrm{H}} 6.07\left(\mathrm{~s}, 2 \mathrm{H},-\mathrm{OCH}_{2} \mathrm{O}-\right) ; 6.99$ (d, $1 \mathrm{H}, J=7.6 \mathrm{~Hz}, \mathrm{Ar}-\mathrm{H})$; 7.35-7.40 (m, $1 \mathrm{H}, \mathrm{Ar}-\mathrm{H})$; 7.45-7.50 (m, 1H, Ar-H); 7.61-7.65 (m, 2H, Ar-H); $7.91(\mathrm{~d}, 1 \mathrm{H}, J=8.4 \mathrm{~Hz}, \operatorname{Ar}-\mathrm{H}) ; 8.05(\mathrm{~d}, 1 \mathrm{H}, J=$ $8.4 \mathrm{~Hz}, \mathrm{Ar}-\mathrm{H}) .{ }^{13} \mathrm{C}$ NMR $\left(125 \mathrm{MHz}, \mathrm{CDCl}_{3}\right) \delta_{\mathrm{C}} 101.5$, 107.4, 108.5, 121.4, 122.4, 123.0, 125.0, 126.3, 128.0, 135.0, 148.5, 150.2, 154.2, 167.5. MS (EI): $m / z=256$ $[\mathrm{M}+\mathrm{H}]^{+}$. Anal. Calcd for $\mathrm{C}_{14} \mathrm{H}_{9} \mathrm{NO}_{2} \mathrm{~S}: \mathrm{C}, 65.87 ; \mathrm{H}$, 3.55 ; N, 5.49\%. Found: C, 66.01; H, 3.51; N, 5.42\%.

2.2q 2-(Phenylbenzo[d]thiazole (2q): Colourless solid; mp $114-116^{\circ} \mathrm{C} ; R_{f}=0.60$ (AcOEt/petroleum ether 10\%). IR (KBr): 3064, 1588, 1555, 1509, 1478, 1433, 1244, 962, $766 \mathrm{~cm}^{-1}$. ${ }^{1} \mathrm{H}$ NMR $(500 \mathrm{MHz}$, $\left.\mathrm{CDCl}_{3}\right) \delta_{\mathrm{H}} 7.38(\mathrm{~d}, 2 \mathrm{H}, J=7.6 \mathrm{~Hz}, \mathrm{Ar}-\mathrm{H}) ; 7.50-755$ (m, 4H, Ar-H); 7.92 (d, 1H, $J=7.6 \mathrm{~Hz}, \mathrm{Ar}-\mathrm{H}) ; 8.07-$ $8.15(\mathrm{~m}, 3 \mathrm{H}, \mathrm{Ar}-\mathrm{H}) .{ }^{13} \mathrm{C}$ NMR $\left(125 \mathrm{MHz}, \mathrm{CDCl}_{3}\right) \delta_{\mathrm{C}}$ $121.5,123.1,125.1,126.2,127.5,129.0,130.8,133.6$, 135.1, 154.2, 167.9. MS (EI): $m / z=211\left[\mathrm{M}^{+}\right]$. Anal. Calcd for $\mathrm{C}_{13} \mathrm{H}_{9} \mathrm{NS}$ : C, 73.90; H, 4.29; N, 6.63\%. Found: C, 74.05; H, 4.24; N, 6.55\%.

2.2r 2-(Pyridin-2-yl)benzo[d]thiazole (2r): Yellow solid; $\mathrm{mp} 130-131^{\circ} \mathrm{C} ; R_{f}=0.15$ (AcOEt/petroleum ether 40\%). IR (KBr): 3322, 3079, 2901, 1655, 989, $874 \mathrm{~cm}^{-1} .{ }^{1} \mathrm{H}$ NMR $\left(500 \mathrm{MHz}, \mathrm{CDCl}_{3}\right) \delta_{\mathrm{H}} 7.35-7.41$ (m, 2H, Ar-H); 7.50-7.54 (m, 1H, Ar-H); 7.81 (ddd, $1 \mathrm{H}, J=1.6,7.6,7.6 \mathrm{~Hz}, \mathrm{Ar}-\mathrm{H}) ; 7.95(\mathrm{dd}, 1 \mathrm{H}, J=$ 
$0.8,8.4 \mathrm{~Hz}, \mathrm{Ar}-\mathrm{H}) ; 8.10(\mathrm{dd}, 1 \mathrm{H}, J=0.8,8.4 \mathrm{~Hz}, \mathrm{Ar}-$ $\mathrm{H})$; $8.35-8.38(\mathrm{~m}, 1 \mathrm{H}, \mathrm{Ar}-\mathrm{H}) ; 8.69(\mathrm{~d}, 1 \mathrm{H}, J=4.7 \mathrm{~Hz}$, Ar-H). ${ }^{13} \mathrm{C}$ NMR $\left(125 \mathrm{MHz}, \mathrm{CDCl}_{3}\right) \delta_{\mathrm{C}} 120.6,121.9$, $123.5,125.2,125.5,126.4,137.1,149.5,151.3,154.1$, 159.0, 169.1. MS (EI): $m / z=213[\mathrm{M}+\mathrm{H}]^{+}$. Anal. Calcd for $\mathrm{C}_{12} \mathrm{H}_{8} \mathrm{~N}_{2} \mathrm{~S}$ : C, 67.90; H, 3.80; N, $13.20 \%$. Found: C, 68.10; H, 3.76; N, 13.11\%.

2.2s 2-(Thiophen-2-yl)benzo[d]thiazole (2s): Colourless solid; $\mathrm{mp} 98-100^{\circ} \mathrm{C} ; R_{f}=0.65$ (AcOEt/ petroleum ether 15\%). IR (KBr): 3083, 3043, 1628, $1064,829 \mathrm{~cm}^{-1}$. ${ }^{1} \mathrm{H}$ NMR $\left(500 \mathrm{MHz}, \mathrm{CDCl}_{3}\right) \delta_{\mathrm{H}} 7.30$ (t, $1 \mathrm{H}, J=3.9 \mathrm{~Hz}$, Ar-H); 7.52-7.63 (m, 2H, Ar-H); $7.65(\mathrm{~d}, 1 \mathrm{H}, J=3.9 \mathrm{~Hz}, \operatorname{Ar}-\mathrm{H}) ; 7.72(\mathrm{~d}, 1 \mathrm{H}, J=$ $3.9 \mathrm{~Hz}) ; 8.10(\mathrm{~d}, 1 \mathrm{H}, J=7.6 \mathrm{~Hz}, \operatorname{Ar}-\mathrm{H}) ; 8.20(\mathrm{~d}, 1 \mathrm{H}$, $J=7.6 \mathrm{~Hz}, \mathrm{Ar}-\mathrm{H}) .{ }^{13} \mathrm{C} \mathrm{NMR}\left(125 \mathrm{MHz}, \mathrm{CDCl}_{3}\right) \delta_{\mathrm{C}}$ 121.3, 123.0, 125.1, 126.3, 127.9, 128.5, 129.2, 134.5, 137.2, 153.5, 161.2. MS (EI): $m / z=217\left[\mathrm{M}^{+}\right]$. Anal. Calcd for $\mathrm{C}_{11} \mathrm{H}_{7} \mathrm{NS}_{2}$ : C, 60.80; H, 3.25; N, 6.45\%. Found: C, 60.99; H, 3.21; N, 6.39\%.

2.2t 2-(Furan-2-yl)benzo[d]thiazole (2t): Yellow solid; $\mathrm{mp} 100-102^{\circ} \mathrm{C} ; R_{f}=0.25$ (AcOEt/petroleum ether 30\%). IR (KBr): 2929, 1602, 1475, 1103, $885 \mathrm{~cm}^{-1} .{ }^{1} \mathrm{H}$ NMR $\left(500 \mathrm{MHz}, \mathrm{CDCl}_{3}\right) \delta_{\mathrm{H}} 6.60-6.62$ $(\mathrm{m}, 1 \mathrm{H}) ; 7.20-7.22(\mathrm{~m}, 1 \mathrm{H}, \mathrm{Ar}-\mathrm{H}) ; 7.36-7.42(\mathrm{~m}, 1 \mathrm{H}$, Ar-H); 7.45-7.50 (m, 1H, Ar-H); $7.61(\mathrm{~s}, 1 \mathrm{H}$, Ar$\mathrm{H}) ; 7.93(\mathrm{~d}, 1 \mathrm{H}, J=8.3 \mathrm{~Hz}, \operatorname{Ar}-\mathrm{H}) ; 8.06(\mathrm{~d}, 1 \mathrm{H}$, $J=8.3 \mathrm{~Hz}) \cdot{ }^{13} \mathrm{C}$ NMR $\left(125 \mathrm{MHz}, \mathrm{CDCl}_{3}\right) \delta_{\mathrm{C}} 111.5$, $112.8,121.8,123.5,125.4,126.7,134.6,141.0,154.0$, 157.8. MS (EI): $m / z=202[\mathrm{M}+\mathrm{H}]^{+}$. Anal. Calcd for $\mathrm{C}_{11} \mathrm{H}_{7} \mathrm{NOS}$ : C, 65.65; H, 3.51; N, 6.96\%. Found: C, $65.41 ; \mathrm{H}, 3.56 ; \mathrm{N}, 7.02 \%$.

2.2u 2-(1-Methyl-1H-indol-2-yl)benzo[d]thiazole $(2 \boldsymbol{u})$ : Colourless solid; mp $147-149^{\circ} \mathrm{C} ; R_{f}=0.66$ (AcOEt/petroleum ether 30\%). IR (KBr): 3419, 3051, 1542, 1450, 1345, 1310, 1191, 1150, 975, 787, $751 \mathrm{~cm}^{-1} .{ }^{1} \mathrm{H}$ NMR $\left(500 \mathrm{MHz}, \mathrm{CDCl}_{3}\right) \delta_{\mathrm{H}} 4.31(\mathrm{~s}$, $\left.3 \mathrm{H},-\mathrm{NCH} H_{3}\right) ; 7.17-7.20$ (m, 2H, Ar-H); 7.33-7.44 (m, $3 \mathrm{H}, \operatorname{Ar}-\mathrm{H}) ; 7.48-7.51(\mathrm{~m}, 1 \mathrm{H}, \mathrm{Ar}-\mathrm{H}) ; 7.67(\mathrm{~d}, 1 \mathrm{H}$, $J=8.4 \mathrm{~Hz}, \mathrm{Ar}-\mathrm{H}) ; 7.88(\mathrm{~d}, 1 \mathrm{H}, J=7.6 \mathrm{~Hz}, \mathrm{Ar}-\mathrm{H})$; $8.06\left(\mathrm{~d}, 1 \mathrm{H}, J=8.4 \mathrm{~Hz}\right.$, Ar-H). ${ }^{13} \mathrm{C}$ NMR $(125 \mathrm{MHz}$, $\left.\mathrm{CDCl}_{3}\right) \delta_{\mathrm{C}} 32.4,107.3,110.2,120.6,121.4,121.6$, 123.3, 124.2, 125.4, 126.4, 127.3, 132.3, 134.5, 139.8, 154.3. 160.7. MS (EI): $m / z=264\left[\mathrm{M}^{+}\right]$. Anal. Calcd for $\mathrm{C}_{16} \mathrm{H}_{12} \mathrm{~N}_{2} \mathrm{~S}$ : C, 72.70; H, 4.58; N, 10.60\%. Found: C, $72.81 ; \mathrm{H}, 4.62 ; \mathrm{N}, 10.53 \%$.

$2.2 \mathrm{v}$ 2-[3-(4-Bromophenyl)-1-phenyl-1H-pyrazol4-yl]benzo[d]thiazole (2v): Colourless solid; mp
200-202 ${ }^{\circ} \mathrm{C} ; R_{f}=0.47$ (AcOEt/petroleum ether $25 \%$ ). IR (KBr): 3359, 1637, 1554, 1506, 1406, 1222, 1085, $829,754,684 \mathrm{~cm}^{-1}$. 1H NMR $\left(500 \mathrm{MHz}, \mathrm{CDCl}_{3}\right) \delta_{\mathrm{H}}$ 7.35-7.37 (m, 2H, Ar-H); 7.46-7.51 (m, 3H, Ar-H); $7.58(\mathrm{~d}, 2 \mathrm{H}, J=8.4 \mathrm{~Hz}, \operatorname{Ar}-\mathrm{H}) ; 7.66(\mathrm{~d}, 2 \mathrm{H}, J=$ $8.4 \mathrm{~Hz}, \mathrm{Ar}-\mathrm{H}) ; 7.80$ (d, 3H, $J=8.4 \mathrm{~Hz}, \mathrm{Ar}-\mathrm{H}) ; 8.00$ (d, $1 \mathrm{H}, J=8.4 \mathrm{~Hz}, \mathrm{Ar}-\mathrm{H}) ; 8.59$ (s, $1 \mathrm{H}$, pyrazolyl-H). ${ }^{13} \mathrm{C}$ NMR $\left(125 \mathrm{MHz}, \mathrm{CDCl}_{3}\right) \delta_{\mathrm{C}} 117.2,119.5,121.5$, 122.8, 123.6, 125.1, 126.4, 127.5, 128.7, 129.7, 131.0, $131.2,131.28,131.7,139.3,151.0,153.4,154.2,154.9$. MS (EI): $m / z=431\left[\mathrm{M}^{+}\right], 433\left[\mathrm{M}^{+2}\right]$. Anal. Calcd for $\mathrm{C}_{22} \mathrm{H}_{13} \mathrm{~N}_{3} \mathrm{SBr}$ : C, 61.12; H, 3.26; N, 9.72\%. Found: C, $61.00 ; \mathrm{H}, 3.33 ; \mathrm{N}, 9.77 \%$.

2.2w 2-[3-(4-Ethoxyphenyl)-1-phenyl-1H-pyrazole4-yl]benzo[d]thiazole $(2 w)$ : Pale yellow solid; $\mathrm{mp}$ $152-154^{\circ} \mathrm{C} ; R_{f}=0.50(\mathrm{AcOEt} /$ petroleum ether $30 \%)$. IR (KBr): 3434, 2965, 1613, 1558, 1503, 1247, 1106, $1043,812 \mathrm{~cm}^{-1} .{ }^{1} \mathrm{H}$ NMR $\left(500 \mathrm{MHz}, \mathrm{CDCl}_{3}\right) \delta_{\mathrm{H}} 1.46$ $\left(\mathrm{t}, 3 \mathrm{H} J=7.5 \mathrm{~Hz},-\mathrm{OCH}_{2} \mathrm{CH}_{3}\right) ; 4.10$ (q, $2 \mathrm{H} J=$ $\left.6.8 \mathrm{~Hz},-\mathrm{OCH}_{2} \mathrm{CH}_{3}\right) ; 6.98(\mathrm{~d}, 2 \mathrm{H}, J=8.6 \mathrm{~Hz}, \mathrm{Ar}-\mathrm{H})$; $7.31(\mathrm{q}, 2 \mathrm{H}, J=8.0 \mathrm{~Hz}, \operatorname{Ar}-\mathrm{H}) ; 7.44-7.50(\mathrm{~m}, 3 \mathrm{H}$, Ar-H); 7.62-7.66 (m, 2H, Ar-H); $7.76(\mathrm{~d}, 1 \mathrm{H}, J=$ $8.0 \mathrm{~Hz}, \mathrm{Ar}-\mathrm{H}) ; 7.81$ (d, $2 \mathrm{H}, J=8.6 \mathrm{~Hz}, \mathrm{Ar}-\mathrm{H}) ; 7.99$ (d, $1 \mathrm{H}, J=8.0 \mathrm{~Hz}, \mathrm{Ar}-\mathrm{H}) ; 8.64$ (s, $1 \mathrm{H}$, pyrazolyl-H). ${ }^{13} \mathrm{C}$ NMR $\left(125 \mathrm{MHz}, \mathrm{CDCl}_{3}\right) \delta_{\mathrm{C}} 14.9,63.6,114.5$, $117.4,119.4,121.5,122.6,124.1,124.9,126.2,127.3$, 128.1, 129.7, 131.1, 135.1, 139.5, 152.2, 153.2, 159.9, 163.2. MS (EI): $m / z=397\left[\mathrm{M}^{+}\right]$. Anal. Calcd for $\mathrm{C}_{24} \mathrm{H}_{19} \mathrm{~N}_{3} \mathrm{OS}$ : C, $72.52 ; \mathrm{H}, 4.82 ; \mathrm{N}, 10.57 \%$. Found: C, $72.67 ; \mathrm{H}, 4.75 ; \mathrm{N}, 10.22 \%$.

2.2x 2-[3-(4-Chlorophenyl)-1-phenyl-1H-pyrazol-4yl]benzo[d]thiazole $(\mathbf{2 x})$ : Colourless solid; mp 173$175^{\circ} \mathrm{C} ; R_{f}=0.45$ (AcOEt/petroleum ether $\left.25 \%\right)$. IR (KBr): 3421, 1599, 1502, 1388, 1203, 1067, 932, $823 \mathrm{~cm}^{-1}$. ${ }^{1} \mathrm{H}$ NMR $\left(500 \mathrm{MHz}, \mathrm{CDCl}_{3}\right) \delta_{\mathrm{H}} 7.33-7.37$ (m, 2H, Ar-H); 7.43-7.51 (m, 5H, Ar-H); 7.72 (d, 2H, $J=8.4 \mathrm{~Hz}, \mathrm{Ar}-\mathrm{H}) ; 7.79=7.82(\mathrm{~m}, 3 \mathrm{H}, \mathrm{Ar}-\mathrm{H}) ; 8.00$ (d, $1 \mathrm{H}, J=8.4 \mathrm{~Hz}, \mathrm{Ar}-\mathrm{H}) ; 8.59$ (s, 1H, pyrazolyl-H). ${ }^{13} \mathrm{C}$ NMR $\left(125 \mathrm{MHz}, \mathrm{CDCl}_{3}\right) \delta_{\mathrm{C}} 117.3,119.4,121.5$, $122.8,125.1,126.4,127.5,128.7,128.8,129.7,130.6$, $131.0,135.0,135.2,139.3,150.9,153.4,159.8$. MS (EI): $m / z=388\left[\mathrm{M}^{+}\right], 390\left[\mathrm{M}^{+2}\right]$. Anal. Calcd for $\mathrm{C}_{22} \mathrm{H}_{14} \mathrm{ClN}_{3} \mathrm{~S}$ : C, 68.12; H, 3.64; N, 10.83\%. Found: C, $67.99 ; \mathrm{H}, 3.76 ; \mathrm{N}, 10.90 \%$.

2.2y 2-[3-(4-Methoxyphenyl)-1-phenyl-1H-pyrazol-4yl]benzo[d]thiazole (2y): Colourless solid; mp 167$169^{\circ} \mathrm{C} ; R_{f}=0.44$ (AcOEt/petroleum ether $30 \%$ ). IR (KBr): 3402, 2346, 1609, 1558, 1505, 1406, 1248, 
1034, 833, $755 \mathrm{~cm}^{-1} \cdot{ }^{1} \mathrm{H}$ NMR $\left(500 \mathrm{MHz}, \mathrm{CDCl}_{3}\right) \delta_{\mathrm{H}}$ $3.87\left(\mathrm{~s}, 3 \mathrm{H},-\mathrm{OCH}_{3}\right) ; 6.99(\mathrm{~d}, 2 \mathrm{H}, J=8.4 \mathrm{~Hz}, \mathrm{Ar}-\mathrm{H})$; $7.32(\mathrm{q}, 2 \mathrm{H}, J=7.6 \mathrm{~Hz}, \mathrm{Ar}-\mathrm{H}) ; 7.44-7.48$ (m, 3H, Ar$\mathrm{H}) ; 7.66(\mathrm{~d}, 2 \mathrm{H}, J=8.4 \mathrm{~Hz}, \operatorname{Ar}-\mathrm{H}) ; 7.76(\mathrm{~d}, 1 \mathrm{H}, J=$ $7.6 \mathrm{~Hz}, \mathrm{Ar}-\mathrm{H}) ; 7.81$ (d, 2H, $J=7.6 \mathrm{~Hz}, \mathrm{Ar}-\mathrm{H}) ; 7.99$ (d, $1 \mathrm{H}, J=8.4 \mathrm{~Hz}, \mathrm{Ar}-\mathrm{H}) ; 8.63$ (s, $1 \mathrm{H}$, pyrazolyl-H). ${ }^{13} \mathrm{C}$ NMR $\left(125 \mathrm{MHz}, \mathrm{CDCl}_{3}\right) \delta_{\mathrm{C}} 55.4,114.0,117.4,119.4$, $121.5,122.6,124.3,124.9,126.2,127.3,128.2$, 129.7, $131.1,135.1,139.5,152.1,153.2,160.4,160.5$. MS (EI): $m / z=383\left[\mathrm{M}^{+}\right]$. Anal. Calcd for $\mathrm{C}_{23} \mathrm{H}_{17} \mathrm{~N}_{3} \mathrm{SO}$ : C, 72.02; H, 4.47; N, 10.96\%. Found: C, 71.89; H, $4.45 ; \mathrm{N}, 11.01 \%$.

$2.2 \mathrm{z}$ 5,6-Dimethoxy-2-phenylbenzo[d]thiazole (2z): Colourless solid; mp $143-145^{\circ} \mathrm{C} ; \quad R_{f}=0.45$ (AcOEt/petroleum ether 10\%). IR (KBr): 3025, 2997, 2837, 1606, 1525, 1491, 1260, $840 \mathrm{~cm}^{-1} .{ }^{1} \mathrm{H}$ NMR $\left(500 \mathrm{MHz}, \mathrm{CDCl}_{3}\right) \delta_{\mathrm{H}} 3.91\left(\mathrm{~s}, 3 \mathrm{H},-\mathrm{OCH}_{3}\right) ; 3.93(\mathrm{~s}$, $\left.3 \mathrm{H},-\mathrm{OCH}_{3}\right) ; 7.55-7.59(\mathrm{~m}, 3 \mathrm{H}, \mathrm{Ar}-\mathrm{H}) ; 7.65(\mathrm{~s}, 1 \mathrm{H}$, Ar-H); 7.72 (s, 1H, Ar-H); 8.06-8.09 (m, 2H, Ar-H). ${ }^{13} \mathrm{C}$ NMR $\left(125 \mathrm{MHz}, \mathrm{CDCl}_{3}\right) \delta_{\mathrm{C}} 56.1,56.3,103.6$, 105.9, 126.6, 127.0, 129.8, 131.1, 133.5, 148.1, 149.0, 165.1. MS (EI): $m / z=271\left[\mathrm{M}^{+}\right]$. Anal. Calcd for $\mathrm{C}_{15} \mathrm{H}_{13} \mathrm{NO}_{2} \mathrm{~S}$ : C, 66.40; H, 4.83; N, 5.16\%. Found: C, $66.19 ; \mathrm{H}, 4.85 ; \mathrm{N}, 6.20 \%$.

\subsection{General procedure for the synthesis of benzoxazoles $2 a^{\prime}-2 z^{\prime}$}

To a pyrex reaction vessel were added 2-aminophenol (1.1 mmol), aldehyde (1.0 mmol), PIFA (1.05 mmol) in ethanol $(3 \mathrm{ml})$. The reaction vessel was then placed in the Emrys Optimizer and exposed to microwave irradiation $\left(80^{\circ} \mathrm{C}\right)$ for $15 \mathrm{~min}$. The reaction mixture was then allowed to cool at room temperature and quenched with $15 \mathrm{~mL}$ of water. The crude reaction mixture was extracted with EtOAc $(3 \times 15 \mathrm{~mL})$. The combined organic layers were dried over anhydrous $\mathrm{Na}_{2} \mathrm{SO}_{4}$, filtered, concentrated and purified by column chromatography on silica gel using petroleum ether/EtOAc to afford the pure product.

2.3a 5-Methyl-2-(2-nitrophenyl)benzo[d]oxazole $\left(2 a^{\prime}\right)$ : Pink solid; mp $134-136^{\circ} \mathrm{C} ; \quad R_{f}=0.49$ (AcOEt/petroleum ether 30\%). IR (KBr): 3431, 2915, 1542, 1480, 1374, 1196, 1044, 800, $772 \mathrm{~cm}^{-1} .{ }^{1} \mathrm{H}$ $\operatorname{NMR}\left(500 \mathrm{MHz}, \mathrm{CDCl}_{3}\right) \delta_{\mathrm{H}} 2.48\left(\mathrm{~s}, 3 \mathrm{H},-\mathrm{CH}_{3}\right) ; 7.19$ $(\mathrm{d}, 1 \mathrm{H}, J=8.4 \mathrm{~Hz}, \mathrm{Ar}-\mathrm{H}) ; 7.42(\mathrm{~d}, 1 \mathrm{H}, J=8.4 \mathrm{~Hz}$, Ar-H); 7.58 (s, 1H, Ar-H); 7.65 (t, $1 \mathrm{H}, J=7.6 \mathrm{~Hz}$, $\operatorname{Ar}-\mathrm{H}) ; 7.71(\mathrm{t}, 1 \mathrm{H}, J=7.6 \mathrm{~Hz}, \operatorname{Ar}-\mathrm{H}) ; 7.86(\mathrm{~d}, 1 \mathrm{H}, J$ $=7.6 \mathrm{~Hz}, \mathrm{Ar}-\mathrm{H}) ; 8.11(\mathrm{~d}, 1 \mathrm{H}, J=7.6 \mathrm{~Hz}, \mathrm{Ar}-\mathrm{H}) .{ }^{13} \mathrm{C}$ NMR $\left(125 \mathrm{MHz}, \mathrm{CDCl}_{3}\right) \delta_{\mathrm{C}} 21.6,110.4,120.5,121.6$,
124.2, 127.3, 131.8, 132.4, 134.9, 141.7, 149.2, 149.3, 158.9. MS (EI): $m / z=254\left[\mathrm{M}^{+}\right]$. Anal. Calcd for $\mathrm{C}_{14} \mathrm{H}_{10} \mathrm{~N}_{2} \mathrm{O}_{3}$ : C, 66.14; H, 3.96; N, 11.02\%. Found: C, $66.00 ; \mathrm{H}, 4.02 ; \mathrm{N}, 10.89 \%$.

2.3b Methyl 4-(benzo[d]oxazol-2-yl)benzoate $\left(\mathbf{2} \boldsymbol{b}^{\prime}\right)$ : Colourless solid; mp: $194-196^{\circ} \mathrm{C} ; \quad R_{f}=0.25$ (AcOEt/petroleum ether 50\%). IR (KBr): 3091, 2925, 2852, 1725, 1606, 740, $707 \mathrm{~cm}^{-1} \cdot{ }^{1} \mathrm{H}$ NMR $(500 \mathrm{MHz}$, $\left.\mathrm{CDCl}_{3}\right) \delta_{\mathrm{H}} 3.97\left(\mathrm{~s}, 3 \mathrm{H},-\mathrm{COOCH}_{3}\right) ; 7.35-7.43(\mathrm{~m}, 2 \mathrm{H}$, Ar-H); 7.60-7.63 (m, 1H, Ar-H); 7.79-7.81 (m, 1H, Ar-H); 8.20 (d, 2H, $J=8.4 \mathrm{~Hz}, \operatorname{Ar}-\mathrm{H}) ; 8.35$ (d, 2H, $J=8.4 \mathrm{~Hz}$, Ar-H). ${ }^{13} \mathrm{C} \mathrm{NMR}\left(125 \mathrm{MHz}, \mathrm{CDCl}_{3}\right) \delta_{\mathrm{C}}$ 52.4, 120.3, 124.9, 125.7, 127.5, 129.5, 130.1, 131.0, 132.6, 141.9, 150.8, 161.9, 166.3. MS (EI): $m / z=253$ $\left[\mathrm{M}^{+}\right]$. Anal. Calcd for $\mathrm{C}_{15} \mathrm{H}_{11} \mathrm{NO}_{3}: \mathrm{C}, 71.14 ; \mathrm{H}, 4.38$; $\mathrm{N}, 5.53 \%$. Found: C, 70.36; H, 4.33; N, 5.44\%.

2.3c 2-(2-Chlorophenyl)-5-methylbenzo[d]oxazole $\left(2 c^{\prime}\right)$ : Colourless solid; mp $74-76^{\circ} \mathrm{C} ; R_{f}=0.59$ (AcOEt/petroleum ether 25\%). IR (KBr): 2921, 1734, 1590, 1548, 1468, 1423, 1325, 1263, 1194, 1019, 774, $730 \mathrm{~cm}^{-1} \cdot{ }^{1} \mathrm{H}$ NMR $\left(500 \mathrm{MHz}, \mathrm{CDCl}_{3}\right) \delta_{\mathrm{H}} 2.49(\mathrm{~s}$, $\left.3 \mathrm{H},-\mathrm{CH}_{3}\right) ; 7.18(\mathrm{~d}, 1 \mathrm{H}, J=8.4 \mathrm{~Hz}, \mathrm{Ar}-\mathrm{H}) ; 7.38(\mathrm{~m}$, 2H, Ar-H); 7.47 (d, 1H, J = 8.4 Hz, Ar-H); 7.54 (d, $1 \mathrm{H}, J=9.2 \mathrm{~Hz}, \operatorname{Ar}-\mathrm{H}) ; 7.62(\mathrm{~s}, 1 \mathrm{H}, \mathrm{Ar}-\mathrm{H}) ; 8.11(\mathrm{~d}$, $1 \mathrm{H}, J=8.4 \mathrm{~Hz}, \mathrm{Ar}-\mathrm{H}) .{ }^{13} \mathrm{C} \mathrm{NMR}\left(125 \mathrm{MHz}, \mathrm{CDCl}_{3}\right)$ $\delta_{\mathrm{C}} 21.6,110.2,120.4,123.5,126.5,126.8,126.9$, $131.4,131.9,133.5,134.6,141.9,148.9,161.1 . \mathrm{MS}$ (EI): $m / z=245\left[\mathrm{M}^{+}\right], 247\left[\mathrm{M}^{+2}\right]$. Anal. Calcd for $\mathrm{C}_{14} \mathrm{H}_{10}$ ClNO: $\mathrm{C}, 69.00 ; \mathrm{H}, 4.14 ; \mathrm{N}, 5.75 \%$. Found: $\mathrm{C}$, $69.22 ; \mathrm{H}, 4.25 ; \mathrm{N}, 5.88 \%$.

2.3d 2-(4-Chlorophenyl)benzo[d]oxazole (2d'): Colourless solid; mp: $143-145^{\circ} \mathrm{C} ; \quad R_{f}=0.50$ (AcOEt/petroleum ether 10\%). IR (KBr): 2961, 1621, $1439,1245,1092,740 \mathrm{~cm}^{-1}$. ${ }^{1} \mathrm{H}$ NMR $(500 \mathrm{MHz}$, $\left.\mathrm{CDCl}_{3}\right) \delta_{\mathrm{H}} 7.28-7.32(\mathrm{~m}, 2 \mathrm{H}, \mathrm{Ar}-\mathrm{H}) ; 7.40-7.45(\mathrm{~m}, 2 \mathrm{H}$, Ar-H); 7.49-7.53 (m, 1H, Ar-H); 7.66-7.70 (m, 1H, Ar-H); 8.10-8.13 (m, 2H, Ar-H). ${ }^{13} \mathrm{C}$ NMR (125 MHz, $\left.\mathrm{CDCl}_{3}\right) \delta_{\mathrm{C}} 110.5,120.0,124.9,125.1,125.8,128.6$, $130.0,129.1,129.2,137.9,141.9,150.9,161.8$. MS (EI): $m / z=229\left[\mathrm{M}^{+}\right], 231\left[\mathrm{M}^{+2}\right]$. Anal. Calcd for $\mathrm{C}_{13} \mathrm{H}_{6}$ ClNO: $\mathrm{C}, 67.99 ; \mathrm{H}, 3.51 ; \mathrm{N}, 6.10 \%$. Found: C, $67.81 ; \mathrm{H}, 3.56 ; \mathrm{N}, 6.18 \%$.

$2.3 \mathrm{e} \quad 2$-p-Tolylbenzo[d]oxazole $\quad\left(2 \boldsymbol{e}^{\prime}\right)$ : Colourless solid; mp: $115-117^{\circ} \mathrm{C} ; R_{f}=0.50($ AcOEt/petroleum ether $10 \%)$. IR (KBr): 3088, 1628, 1244, $1055 \mathrm{~cm}^{-1}$. ${ }^{1} \mathrm{H}$ NMR $\left(500 \mathrm{MHz}, \mathrm{CDCl}_{3}\right) \delta_{\mathrm{H}} 2.41\left(\mathrm{~s}, 3 \mathrm{H},-\mathrm{CH}_{3}\right)$; 6.83-7.16 (4H, m, Ar-H); 7.35-7.81 (m, 4H, Ar-H). 
${ }^{13} \mathrm{C}$ NMR $\left(125 \mathrm{MHz}, \mathrm{CDCl}_{3}\right) \delta_{\mathrm{C}} 22.4,110.6,120.8$, $123.5,125.5,126.8,127.9,131.1,142.2,150.9,164.7$. MS (EI): $m / z=209\left[\mathrm{M}^{+}\right]$. Anal. Calcd for $\mathrm{C}_{14} \mathrm{H}_{11} \mathrm{NO}$ : C, 80.36; H, 5.30; N, 6.69\%. Found: C, 80.12; H, 5.35; $\mathrm{N}, 6.77 \%$.

$2.3 \mathrm{f}$ 2-(3,4,5-Trimethoxyphenyl)benzo[d]oxazole $\left(2 f^{\prime}\right)$ : Colourless solid; $\mathrm{mp} 111-113^{\circ} \mathrm{C} ; R_{f}=0.25$ (AcOEt/petroleum ether 10\%). IR (KBr): 2935, 1632, 1246, 1145, $1058 \mathrm{~cm}^{-1} .{ }^{1} \mathrm{H}$ NMR $\left(500 \mathrm{MHz}, \mathrm{CDCl}_{3}\right)$ $\delta_{\mathrm{H}} 3.89\left(\mathrm{~s}, 3 \mathrm{H},-\mathrm{OCH}_{3}\right) ; 3.91\left(\mathrm{~s}, 3 \mathrm{H},-\mathrm{OCH}_{3}\right) ; 3.96(\mathrm{~s}$, $\left.3 \mathrm{H},-\mathrm{OCH}_{3}\right) ; 7.35-7.69$ (m, 4H, Ar-H) 7.99-8.18 (m, $2 \mathrm{H}, \mathrm{Ar}-\mathrm{H}) .{ }^{13} \mathrm{C} \mathrm{NMR}\left(125 \mathrm{MHz}, \mathrm{CDCl}_{3}\right) \delta_{\mathrm{C}} 57.0,57.5$, 58.7, 112.8, 122.7, 124.4, 127.5, 131.0, 132.5, 143.4, 149.5, 150.1, 151.1. MS (EI): $m / z=285\left[\mathrm{M}^{+}\right]$. Anal. Calcd for $\mathrm{C}_{16} \mathrm{H}_{15} \mathrm{NO}_{4}$ : C, 67.36; $\mathrm{H}, 5.30 ; \mathrm{N}, 4.91 \%$. Found: C, 67.51; H, 5.26; N, 4.85\%.

2.3g Methyl 3-(benzo[d]oxazol-2-yl)benzoate (2 $\left.\mathbf{g}^{\prime}\right)$ : Yellow solid; mp: $128-130^{\circ} \mathrm{C} ; \quad R_{f}=0.30$ (AcOEt/petroleum ether 50\%). IR (KBr): 3083, 2950, 2922, 1720, 1606, $745 \mathrm{~cm}^{-1}$. ${ }^{1} \mathrm{H}$ NMR $(500 \mathrm{MHz}$, $\left.\mathrm{CDCl}_{3}\right) \delta_{\mathrm{H}} 3.96\left(\mathrm{~s}, 3 \mathrm{H},-\mathrm{COOCH}_{3}\right) ; 7.40-7.43(\mathrm{~m}$, 2H, Ar-H); 7.60-7.65 (m, 2H, Ar-H); 7.80-7.83 (m, $1 \mathrm{H}, \operatorname{Ar}-\mathrm{H}) ; 8.25(\mathrm{~d}, 1 \mathrm{H}, J=7.6 \mathrm{~Hz}, \operatorname{Ar}-\mathrm{H}) ; 8.48(\mathrm{~d}$, $1 \mathrm{H}, J=7.6 \mathrm{~Hz}, \mathrm{Ar}-\mathrm{H}) ; 8.95$ (s, $1 \mathrm{H}, \mathrm{Ar}-\mathrm{H}) .{ }^{13} \mathrm{C}$ NMR $\left(125 \mathrm{MHz}, \mathrm{CDCl}_{3}\right) \delta_{\mathrm{C}} 110.7,120.1,124.9,125.5$, 127.4, 128.7, 129.2, 131.1, 131.7, 132.5, 141.7, 150.7, 162.0, 166.2. MS (EI): $m / z=253\left[\mathrm{M}^{+}\right]$. Anal. Calcd for $\mathrm{C}_{15} \mathrm{H}_{11} \mathrm{NO}_{3}$ : C, 71.14; $\mathrm{H}, 4.38 ; \mathrm{N}, 5.53 \%$. Found: C, 70.99; H, 4.41; N, 5.60\%.

2.3h 5-Methyl-2-(4-nitrophenyl)benzo[d]oxazole $\left(2 h^{\prime}\right)$ : Pale yellow solid; mp $218-250^{\circ} \mathrm{C} ; R_{f}=0.44$ (AcOEt/petroleum ether 30\%). IR (KBr): 3402, 1556, 1521, 1342, 854, $706 \mathrm{~cm}^{-1}$. ${ }^{1} \mathrm{H}$ NMR $(500 \mathrm{MHz}$, $\left.\mathrm{CDCl}_{3}\right) \delta_{\mathrm{H}} 2.50\left(\mathrm{~s}, 3 \mathrm{H},-\mathrm{CH}_{3}\right) ; 7.22(\mathrm{~d}, 1 \mathrm{H}, J=8.4 \mathrm{~Hz}$, $\mathrm{Ar}-\mathrm{H}) ; 7.48(\mathrm{~d}, 1 \mathrm{H}, J=8.4 \mathrm{~Hz}$ Ar-H); $7.59(\mathrm{~s}, 1 \mathrm{H}$, Ar-H); 8.35-8.41 (m, 4H, Ar-H). ${ }^{13} \mathrm{C}$ NMR $(125 \mathrm{MHz}$, $\left.\mathrm{CDCl}_{3}\right) \delta_{\mathrm{C}} 21.6,110.4,120.5,124.3,127.6,128.4$, 133.0, 135.3, 142.2, 149.4, 160.8, 162.7. MS (EI): $m / z=254\left[\mathrm{M}^{+}\right]$. Anal. Calcd for $\mathrm{C}_{14} \mathrm{H}_{10} \mathrm{~N}_{2} \mathrm{O}_{3}: \mathrm{C}$, 66.14; H, 3.96; N, 11.02\%. Found: C, 66.32; H, 4.10; $\mathrm{N}, 10.92 \%$.

2.3i 5-Nitro-2-phenylbenzo[d]oxazole (2i'): Yellow solid; mp: $241-243^{\circ} \mathrm{C} ; R_{f}=0.20(\mathrm{AcOEt} /$ petroleum ether 30\%). IR (KBr): 1641, 1525, 1350, 1247, $1051 \mathrm{~cm}^{-1} .{ }^{1} \mathrm{H}$ NMR $\left(500 \mathrm{MHz}, \mathrm{CDCl}_{3}\right) \delta_{\mathrm{H}} 7.51-7.58$ $(\mathrm{m}, 3 \mathrm{H}, \operatorname{Ar}-\mathrm{H}) ; 7.70(\mathrm{~d}, 1 \mathrm{H}, J=9.2 \mathrm{~Hz}, \operatorname{Ar}-\mathrm{H})$; 8.22-8.27 (m, 2H, Ar-H); 8.29 (dd, $1 \mathrm{H}, J=9.2$,
$2.1 \mathrm{~Hz}, \operatorname{Ar}-\mathrm{H}) ; 8.65(\mathrm{~d}, 2 \mathrm{H}, J=2.1 \mathrm{~Hz}, \mathrm{Ar}-\mathrm{H}) \cdot{ }^{13} \mathrm{C}$ NMR $\left(125 \mathrm{MHz}, \mathrm{CDCl}_{3}\right) \delta_{\mathrm{C}} 110.8,116.1,121.0,125.8$, 127.9, 129.0, 132.5, 142.5, 154.0. MS (EI): $\mathrm{m} / z=240$ $\left[\mathrm{M}^{+}\right]$. Anal. Calcd for $\mathrm{C}_{13} \mathrm{H}_{8} \mathrm{~N}_{2} \mathrm{O}_{3}: \mathrm{C}, 65.00 ; \mathrm{H}, 3.36$; N, $11.66 \%$. Found: C, 64.81; H, 3.41; N, $11.75 \%$.

2.3j 2-(4-Bromophenyl)benzo[d]oxazole $\left(2 j^{\prime}\right)$ : Pale yellow solid; mp: $142-144^{\circ} \mathrm{C} ; R_{f}=0.40$ (AcOEt/petroleum ether 25\%). IR (KBr): 1581, 1501, 1269, 1052, 944, 830, $799 \mathrm{~cm}^{-1} .{ }^{1} \mathrm{H}$ NMR $(500 \mathrm{MHz}$, $\left.\mathrm{CDCl}_{3}\right) \delta_{\mathrm{H}} 7.30-7.37(\mathrm{~m}, 2 \mathrm{H}, \mathrm{Ar}-\mathrm{H}) ; 7.50-7.55(\mathrm{~m}, 1 \mathrm{H}$, Ar-H); 7.60-7.65 (m, 2H, Ar-H); 7.70-7.75 (m, 1H, Ar-H); 8.05-8.10 (m, 2H, Ar-H). ${ }^{13} \mathrm{C}$ NMR $(125 \mathrm{MHz}$, $\left.\mathrm{CDCl}_{3}\right) \delta_{\mathrm{C}} 110.5,120.0,124.6,125.5,126.1,126.3$, 128.9, 132.3, 142.0, 150.5, 161.9. MS (EI): $m / z=$ $273\left[\mathrm{M}^{+}\right], 275\left[\mathrm{M}^{+2}\right]$. Anal. Calcd for $\mathrm{C}_{13} \mathrm{H}_{8}$ BrNO: $\mathrm{C}$, 56.96; H, 2.94; N, 5.11\%. Found: C, 57.16; H, 2.90; N, $5.05 \%$.

2.3k 2-(4-Nitrophenyl)benzo[d]oxazole $\left(2 \boldsymbol{k}^{\prime}\right)$ : Yellow solid; mp: $262-264^{\circ} \mathrm{C} ; \quad R_{f}=0.25$ (AcOEt/petroleum ether 50\%). IR (KBr): 1640, 1524, 1346, 1242, $1058 \mathrm{~cm}^{-1} .{ }^{1} \mathrm{H}$ NMR $\left(500 \mathrm{MHz}, \mathrm{CDCl}_{3}\right)$ $\delta_{\mathrm{H}} 7.75-8.11(\mathrm{~m}, 4 \mathrm{H}, \mathrm{Ar}-\mathrm{H}) ; 8.22-8.5(4 \mathrm{H}, \mathrm{m}, \mathrm{Ar}-$ $\mathrm{H}) .{ }^{13} \mathrm{C}$ NMR $\left(125 \mathrm{MHz}, \mathrm{CDCl}_{3}\right) \delta_{\mathrm{C}} 112.4,120.8$, 124.8, 126.0, 127.0, 129.1, 130.7, 141.0, 148.9, 150.5, 161.0. MS (EI): $m / z=240\left[\mathrm{M}^{+}\right]$. Anal. Calcd for $\mathrm{C}_{13} \mathrm{H}_{8} \mathrm{~N}_{2} \mathrm{O}_{3}$ : C, 65.00; H, 3.36; N, 11.66\%. Found: C, $65.13 ; \mathrm{H}, 3.31 ; \mathrm{N}, 11.58 \%$.

2.31 2-(Benzo[d][1,3]dioxol-5-yl)benzo[d]oxazole $\left(2 l^{\prime}\right)$ : Yellow solid; mp $147-149^{\circ} \mathrm{C} ; \quad R_{f}=0.30$ (AcOEt/petroleum ether 20\%). IR (KBr): 2931, 1639, 1245, 1042, 1115, $761 \mathrm{~cm}^{-1}$. ${ }^{1} \mathrm{H}$ NMR $(500 \mathrm{MHz}$, $\left.\mathrm{CDCl}_{3}\right) \delta_{\mathrm{H}} 4.39\left(\mathrm{~s}, 2 \mathrm{H},-\mathrm{OCH} \mathrm{H}_{2} \mathrm{O}-\right) ; 6.86-7.02(\mathrm{~m}, 3 \mathrm{H}$, $\mathrm{Ar}-\mathrm{H})$; 7.37-7.78 (m, 4H, Ar-H). ${ }^{13} \mathrm{C}$ NMR (125 MHz, $\left.\mathrm{CDCl}_{3}\right) \delta_{\mathrm{C}} 91.1,113.0,114.5,119.0,124.8,125.5$, 131.0, 138.4, 145.2, 146.7. MS (EI): $m / z=239\left[\mathrm{M}^{+}\right]$. Anal. Calcd for $\mathrm{C}_{14} \mathrm{H}_{9} \mathrm{NO}_{3}: \mathrm{C}, 70.29 ; \mathrm{H}, 3.79 ; \mathrm{N}$, 5.86\%. Found: C, 70.51; H, 3.66; N, 5.79\%.

$2.3 \mathrm{~m}$ 2-(4-Methoxyphenyl)benzo[d]oxazole $\left(2 \mathrm{~m}^{\prime}\right)$ : Colourless solid; mp: $97-99^{\circ} \mathrm{C} ; \quad R_{f}=0.55$ (AcOEt/petroleum ether 10\%). IR (KBr): 3052, 1618, 1255, 1248, 1037, 1025, $802 \mathrm{~cm}^{-1}$. ${ }^{1} \mathrm{H}$ NMR $(500 \mathrm{MHz}$, $\left.\mathrm{CDCl}_{3}\right) \delta_{\mathrm{H}} 3.88\left(\mathrm{~s}, 3 \mathrm{H},-\mathrm{OCH}_{3}\right) ; 7.00(\mathrm{~d}, 2 \mathrm{H}, J=$ 8.4 Hz, Ar-H); 7.29-7.36 (m, 2H, Ar-H); 7.48-7.52 (m, 1H, Ar-H); 7.65-7.73 (m, 1H, Ar-H); 8.21 (d, 2H, $J=$ $8.7 \mathrm{~Hz}, \mathrm{Ar}-\mathrm{H}) .{ }^{13} \mathrm{C} \mathrm{NMR}\left(125 \mathrm{MHz}, \mathrm{CDCl}_{3}\right) \delta_{\mathrm{C}} 55.5$, $110.5,114.5,119.5,119.9,124.5,124.8,129.4,142.3$, 150.8, 162.3, 162.8. MS (EI): $m / z=225\left[\mathrm{M}^{+}\right]$. Anal. 
Calcd for $\mathrm{C}_{14} \mathrm{H}_{11} \mathrm{NO}_{2}$ : C, 74.65; H, 4.92; N, 6.22\%. Found: C, 74.81; H, 4.89; N, 6.15\%.

2.3n 2-(3,4-Dichlorophenyl)benzo[d]oxazole $\quad\left(\mathbf{2} \boldsymbol{n}^{\prime}\right)$ : Colourless solid; mp: $139-141^{\circ} \mathrm{C} ; \quad R_{f}=0.50$ (AcOEt/petroleum ether 30\%). IR (KBr): 2963, 1620, 1440, 1241, 1099, $747 \mathrm{~cm}^{-1}$. ${ }^{1} \mathrm{H}$ NMR $(500 \mathrm{MHz}$, $\left.\mathrm{CDCl}_{3}\right) \delta_{\mathrm{H}} 7.20-7.24(\mathrm{~m}, 2 \mathrm{H}$, Ar-H); 7.31-7.39 (m, 2H, Ar-H); 7.55-7.63 (m, 1H, Ar-H); 7.75-7.80 (m, $1 \mathrm{H}, \operatorname{Ar}-\mathrm{H}) ; 8.05(\mathrm{dt}, 1 \mathrm{H}, J=8.1,2.1 \mathrm{~Hz}, \operatorname{Ar}-\mathrm{H})$; $8.34(\mathrm{t}, 1 \mathrm{H}, J=2.1 \mathrm{~Hz}, \mathrm{Ar}-\mathrm{H}) .{ }^{13} \mathrm{C}$ NMR $(125 \mathrm{MHz}$, $\left.\mathrm{CDCl}_{3}\right) \delta_{\mathrm{C}} 110.5,120.0,125.0,125.6,126.3,127.0$, $129.2,131.0,133.3,141.6,150.5,160.9$. MS (EI): $m / z=263\left[\mathrm{M}^{+}\right], 265\left[\mathrm{M}^{+2}\right], 267\left[\mathrm{M}^{+4}\right]$. Anal. Calcd for $\mathrm{C}_{13} \mathrm{H}_{7} \mathrm{Cl}_{2} \mathrm{NO}$ : C, 59.12; H, 2.67; N, 5.30\%. Found: C, 58.96; H, 2.71; N, 5.37\%.

2.3o 2-(3-Methoxyphenyl)benzo[d]oxazole $\quad\left(\mathbf{2} \boldsymbol{o}^{\prime}\right)$ : Yellow solid; $\mathrm{mp}: \quad 107-109^{\circ} \mathrm{C} ; \quad R_{f}=0.50$ (AcOEt/petroleum ether 10\%). IR (KBr): 3055, 1620, 1250, 1241, 1030, $1021 \mathrm{~cm}^{-1}$. ${ }^{1} \mathrm{H}$ NMR $(500 \mathrm{MHz}$, $\left.\mathrm{CDCl}_{3}\right) \delta_{\mathrm{H}} 3.95\left(\mathrm{~s}, 3 \mathrm{H},-\mathrm{OCH}_{3}\right) ; 7.10-7.15(\mathrm{~m}, 1 \mathrm{H}$, Ar-H); 7.35-7.41 (m, 2H, Ar-H); 7.47 (dd, 1H, J = 8.1, 8.1 Hz, Ar-H); 7.60-7.65 (m, 1H, Ar-H); 7.75$7.82(\mathrm{~m}, 2 \mathrm{H}, \mathrm{Ar}-\mathrm{H}) ; 7.85-7.90(\mathrm{~m}, 1 \mathrm{H}, \mathrm{Ar}-\mathrm{H}) .{ }^{13} \mathrm{C}$ NMR $\left(125 \mathrm{MHz}, \mathrm{CDCl}_{3}\right) \delta_{\mathrm{C}} 55.5,110.7,112.1,118.5$, 119.9, 124.4, 125.0, 128.2, 128.9, 141.9, 151.0, 159.9, 162.8. MS (EI): $m / z=225\left[\mathrm{M}^{+}\right]$. Anal. Calcd for $\mathrm{C}_{14} \mathrm{H}_{11} \mathrm{NO}_{2}$ : C, 74.65; H, 4.92; N, 6.22\%. Found: C, 74.52; H, 4.95; N, 6.29\%.

$2.3 p$ 5-Chloro-2-(3-nitrophenyl)benzo[d]oxazole $\left(2 p^{\prime}\right)$ : Colourless solid; mp $184-186^{\circ} \mathrm{C} ; R_{f}=0.52$ (AcOEt/petroleum ether 30\%). IR (KBr): 3424, 2361, $1526,1449,1351,1100,821 \mathrm{~cm}^{-1} .{ }^{1} \mathrm{H}$ NMR $(500 \mathrm{MHz}$, $\left.\mathrm{CDCl}_{3}\right) \delta_{\mathrm{H}} 7.38(\mathrm{q}, 1 \mathrm{H}, J=8.4 \mathrm{~Hz}, \mathrm{Ar}-\mathrm{H}) ; 7.54(\mathrm{~d}, 1 \mathrm{H}$, $J=9.1 \mathrm{~Hz}, \mathrm{Ar}-\mathrm{H}) ; 7.72(\mathrm{~d}, 1 \mathrm{H}, J=8.4 \mathrm{~Hz}, \mathrm{Ar}-\mathrm{H})$; $7.78(\mathrm{~s}, 1 \mathrm{H}, \mathrm{Ar}-\mathrm{H}) ; 8.39$ (d, $1 \mathrm{H}, J=8.8 \mathrm{~Hz}, \operatorname{Ar}-\mathrm{H})$; $8.55(\mathrm{~d}, 1 \mathrm{H}, J=7.6 \mathrm{~Hz}, \operatorname{Ar}-\mathrm{H}) ; 9.07$ (s, $1 \mathrm{H}$, Ar-H). ${ }^{13} \mathrm{C}$ NMR $\left(125 \mathrm{MHz}, \mathrm{CDCl}_{3}\right) \delta_{\mathrm{C}} 111.8,120.5,122.7$, 126.3, 126.5, 130.4, 130.7, 133.3, 142.9, 149.5, 157.5, 161.9. MS (EI): $m / z=274\left[\mathrm{M}^{+}\right], 276\left[\mathrm{M}^{+2}\right]$. Anal. Calcd for $\mathrm{C}_{13} \mathrm{H}_{7} \mathrm{ClN}_{2} \mathrm{O}_{3}$ : C, 56.85; H, 2.57; N, $10.20 \%$. Found: C, 56.75; H, 2.49; N, 10.15\%.

2.3q 2-Phenylbenzo[d]oxazole $\left(2 q^{\prime}\right)$ : Colourless solid; mp: $100-102^{\circ} \mathrm{C} ; R_{f}=0.60(\mathrm{AcOEt} /$ petroleum ether 10\%). IR (KBr): 2975, 1614, 1248, 1040, $803 \mathrm{~cm}^{-1} .{ }^{1} \mathrm{H}$ NMR $\left(500 \mathrm{MHz}, \mathrm{CDCl}_{3}\right) \delta_{\mathrm{H}} 7.35-7.40$ (m, 2H, Ar-H); 7.50-7.55 (m, 2H, Ar-H); 7.60-7.63 $(\mathrm{m}, 1 \mathrm{H}$, Ar-H); 7.75-7.85 (m, 1H, Ar-H); 8.25-8.31 (m, 2H, Ar-H). ${ }^{13} \mathrm{C}$ NMR $\left(125 \mathrm{MHz}, \mathrm{CDCl}_{3}\right) \delta_{\mathrm{C}} 110.5$, 120.0, 124.5, 125.0, 127.0, 127.8, 129.0, 131.6, 142.0, 150.8, 162.9. MS (EI): $m / z=195\left[\mathrm{M}^{+}\right]$. Anal. Calcd for $\mathrm{C}_{13} \mathrm{H}_{9} \mathrm{NO}$ : C, 79.98; H, 4.65; N, 7.17\%. Found: C, $80.11 ; \mathrm{H}, 4.61 ; \mathrm{N}, 7.08 \%$.

\section{3r 2-(2-Chlorophenyl)benzo[d]oxazole $\left(2 \boldsymbol{r}^{\prime}\right)$ :} Colourless solid; $\mathrm{mp}$ 61-64 ${ }^{\circ} \mathrm{C} ; \quad R_{f}=0.53$ (AcOEt/petroleum ether 30\%). IR (KBr): 2953, 1537, 1430, 1253, 1194, 1022, 806, $738 \mathrm{~cm}^{-1}$. ${ }^{1} \mathrm{H}$ NMR $\left(500 \mathrm{MHz}, \mathrm{CDCl}_{3}\right) \delta_{\mathrm{H}} 7.36-7.46(\mathrm{~m}, 4 \mathrm{H}, \quad \mathrm{Ar}-\mathrm{H})$; 7.56-7.57 (m, 1H, Ar-H); 7.61-7.62 (m, 1H, Ar-H); 7.84-7.86 (m, 1H, Ar-H); $8.13(\mathrm{dd}, 1 \mathrm{H}, J=7.6$, $2.3 \mathrm{~Hz}, \mathrm{Ar}-\mathrm{H}) .{ }^{13} \mathrm{C}$ NMR $\left(125 \mathrm{MHz}, \mathrm{CDCl}_{3}\right) \delta_{\mathrm{C}} 110.9$, 120.6, 124.8, 125.7, 126.2, 127.1, 131.5, 131.9, 132.0, 133.6, 141.8, 150.6, 161.1. MS (EI): $m / z=229\left[\mathrm{M}^{+}\right]$, $231\left[\mathrm{M}^{+2}\right]$. Anal. Calcd for $\mathrm{C}_{13} \mathrm{H}_{6} \mathrm{ClNO}$ : C, 67.99; H, $3.51 ; \mathrm{N}, 6.10 \%$. Found: C, 68.11; H, 3.62; N, 5.99\%.

2.3s 2-(Furan-2-yl)benzo[d]oxazole (2s'): Colourless solid; $\mathrm{mp} 90-92^{\circ} \mathrm{C} ; R_{f}=0.45(\mathrm{AcOEt} /$ petroleum ether 15\%). IR (KBr): 2926, 1620, 1245, 1141, 1046, $751 \mathrm{~cm}^{-1}$. ${ }^{1} \mathrm{H}$ NMR $\left(500 \mathrm{MHz}, \mathrm{CDCl}_{3}\right) \delta_{\mathrm{H}} 6.51-6.98$ (m, 3H, Ar-H); 7.02-7.56 (m, 4H, Ar-H). ${ }^{13} \mathrm{C}$ NMR $\left(125 \mathrm{MHz}, \mathrm{CDCl}_{3}\right) \delta_{\mathrm{C}} 103.9,115.0,116.2,124.5$, $125.6,126.2,145.0,146.3,150.5,153.1,156.5$. MS (EI): $m / z=185\left[\mathrm{M}^{+}\right]$. Anal. Calcd for $\mathrm{C}_{11} \mathrm{H}_{7} \mathrm{NO}_{2}: \mathrm{C}$, 71.35; H, 3.81; N, 7.56\%. Found: C, 71.05; H, 3.86; N, $7.65 \%$.

2.3t 2-(Thiophen-2-yl)benzo[d]oxazole (2t'): Yellow solid; $\mathrm{mp} 104-106^{\circ} \mathrm{C} ; R_{f}=0.50$ (AcOEt/ petroleum ether 10\%). IR (KBr): 2930, 1638, 1252, $1135,1043,749 \mathrm{~cm}^{-1} .{ }^{1} \mathrm{H}$ NMR $\left(500 \mathrm{MHz}, \mathrm{CDCl}_{3}\right) \delta_{\mathrm{H}}$ 6.66-6.98 (m, 3H, Ar-H); 7.52-7.72 (m, 4H, Ar-H). ${ }^{13} \mathrm{C}$ NMR $\left(125 \mathrm{MHz}, \mathrm{CDCl}_{3}\right) \delta_{\mathrm{C}} 112.5,120.8,124.5$, 125.0, 126.5, 127.0, 127.2, 138.0, 140.6, 149.1, 154.6. MS (EI): $m / z=201\left[\mathrm{M}^{+}\right]$. Anal. Calcd for $\mathrm{C}_{11} \mathrm{H}_{7} \mathrm{NOS}$ : C, 65.65; H, 3.51; N, 6.96\%. Found: C, 65.83; H, 3.46; $\mathrm{N}, 6.88 \%$.

$2.3 \mathrm{u} \quad 2-\left(1 \mathrm{H}\right.$-Pyrrol-2-yl)benzo[d]oxazole $\left(\mathbf{2} \boldsymbol{u}^{\prime}\right)$ : Pink solid; $\mathrm{mp} 144-146^{\circ} \mathrm{C} ; R_{f}=0.51$ (AcOEt/petroleum ether 30\%). IR (KBr): 3401, 1629, 1585, 1455, 1403, $1243,1117,741 \mathrm{~cm}^{-1} .{ }^{1} \mathrm{H}$ NMR $\left(500 \mathrm{MHz}, \mathrm{CDCl}_{3}\right) \delta_{\mathrm{H}}$ 6.36-6.38 (m, 1H, Ar-H); 7.04-7.05 (m, 1H, Ar-H); 7.28-7.33 (m, 2H, Ar-H); 7.52 (d, $1 \mathrm{H}, J=7.6 \mathrm{~Hz}$, Ar$\mathrm{H}) ; 7.64(\mathrm{~d}, 1 \mathrm{H}, J=7.6 \mathrm{~Hz}, \mathrm{Ar}-\mathrm{H}) ; 10.25(\mathrm{~s}, 1 \mathrm{H},-\mathrm{N} H)$. ${ }^{13} \mathrm{C}$ NMR $\left(125 \mathrm{MHz}, \mathrm{CDCl}_{3}\right) \delta_{\mathrm{C}} 110.5,110.9,113.3$, 118.9, 119.9, 123.1, 124.4, 124.7, 150.2, 158.2, 163.7. MS (EI): $m / z=184\left[\mathrm{M}^{+}\right]$. Anal. Calcd for $\mathrm{C}_{11} \mathrm{H}_{8} \mathrm{~N}_{2} \mathrm{O}$ : 
C, 71.73; H, 4.38; N, 15.21\%. Found: C, 71.81; H, 4.25; N, $15.25 \%$.

$2.3 \mathrm{v} \quad 2-(1-M e t h y l-1 H$-indol-2-yl)benzo[d]oxazole $\left(2 v^{\prime}\right)$ : Colourless solid; mp $161-163^{\circ} \mathrm{C} ; R_{f}=0.55$ (AcOEt/petroleum ether 25\%). IR (KBr): 2332, 1579, 1450, 1340, 1240, 1141, $753 \mathrm{~cm}^{-1} .{ }^{1} \mathrm{H}$ NMR $(500 \mathrm{MHz}$, $\left.\mathrm{CDCl}_{3}\right) \delta_{\mathrm{H}} 4.31\left(\mathrm{~s}, 3 \mathrm{H},-\mathrm{NCH}_{3}\right) ; 7.18(\mathrm{t}, 1 \mathrm{H}, J=$ 7.6 Hz, Ar-H); 7.35-7.38 (m, 3H, Ar-H); 7.42 (d, 2H, $J$ $=10.7 \mathrm{~Hz}, \mathrm{Ar}-\mathrm{H}) ; 7.57(\mathrm{~d}, 1 \mathrm{H}, J=7.6 \mathrm{~Hz}, \mathrm{Ar}-\mathrm{H}) ; 7.72$ (d, $1 \mathrm{H}, J=7.6 \mathrm{~Hz}, \mathrm{Ar}-\mathrm{H}) ; 7.80-780$ (m, 1H, Ar-H). ${ }^{13} \mathrm{C}$ NMR $\left(125 \mathrm{MHz}, \mathrm{CDCl}_{3}\right) \delta_{\mathrm{C}} 32.2,107.6,110.2$, $110.5,119.9,120.7,122.1,124.5,124.6,125.2,126.3$, 126.9, 139.9, 142.2, 149.9, 157.8. MS (EI): $m / z=248$ $\left[\mathrm{M}^{+}\right]$. Anal. Calcd for $\mathrm{C}_{16} \mathrm{H}_{12} \mathrm{~N}_{2} \mathrm{O}: \mathrm{C}, 77.40 ; \mathrm{H}, 4.87$; $\mathrm{N}, 11.28 \%$. Found: C, 77.55; H, 4.75; N, $11.39 \%$.

2.3w 2-[3-(4-Chlorophenyl)-1-phenyl-1H-pyrazol-4yl]benzo[d]oxazole $\left(\mathbf{2} \boldsymbol{w}^{\prime}\right)$ : Colourless solid; mp 205$207^{\circ} \mathrm{C} ; R_{f}=0.44$ (AcOEt/petroleum ether $25 \%$ ). IR (KBr): 3411, 1627, 1590, 1502, 1454, 1391, 1244, $1093,989 \mathrm{~cm}^{-1} .{ }^{1} \mathrm{H}$ NMR $\left(500 \mathrm{MHz}, \mathrm{CDCl}_{3}\right) \delta_{\mathrm{H}}$ 7.32-7.33 (m, 2H, Ar-H); 7.37-7.39 (m, 1H, Ar-H); 7.45-7.53 (m, 5H, Ar-H); $7.71(\mathrm{~d}, 1 \mathrm{H}, J=9.1 \mathrm{~Hz}$, Ar$\mathrm{H}) ; 7.81(\mathrm{~d}, 2 \mathrm{H}, J=8.4 \mathrm{~Hz}, \operatorname{Ar}-\mathrm{H}) ; 7.99(\mathrm{~d}, 2 \mathrm{H}, J=$ $8.4 \mathrm{~Hz}, \mathrm{Ar}-\mathrm{H}) ; 8.71$ (s, $1 \mathrm{H}$, pyrazolyl-H). ${ }^{13} \mathrm{C} \mathrm{NMR}$ $\left(125 \mathrm{MHz}, \mathrm{CDCl}_{3}\right) \delta_{\mathrm{C}} 110.3,110.4,119.5,124.6$, $124.9,127.7,128.5,129.8,130.3,130.6,134.9,139.3$, 141.9, 150.2, 151.1, 158.1. MS (EI): $m / z=372\left[\mathrm{M}^{+}\right]$, $374\left[\mathrm{M}^{+2}\right]$. Anal. Calcd for $\mathrm{C}_{22} \mathrm{H}_{14} \mathrm{ClN}_{3} \mathrm{O}: \mathrm{C}, 71.07 ; \mathrm{H}$, $3.80 ; \mathrm{N}, 11.30 \%$. Found: C, 71.25; H, 3.75; N, $11.25 \%$.

$2.3 x \quad 2-[3-(4-B r o m o p h e n y l)-1-p h e n y l-1 H$-pyrazol-4yl]-5-methylbenzo[d]oxazole $\left(2 \boldsymbol{x}^{\prime}\right)$ : Colourless solid; $\mathrm{mp} 210-214^{\circ} \mathrm{C} ; R_{f}=0.52(\mathrm{AcOEt} /$ petroleum ether $30 \%)$. IR (KBr): 2920, 1589, 1500, 1262, 1223, 1057, 944, 830, $799 \mathrm{~cm}^{-1} .{ }^{1} \mathrm{H}$ NMR $\left(500 \mathrm{MHz}, \mathrm{CDCl}_{3}\right) \delta_{\mathrm{H}}$ $2.46\left(\mathrm{~s}, 3 \mathrm{H},-\mathrm{CH}_{3}\right) ; 7.11(\mathrm{~d}, 1 \mathrm{H}, J=8.4 \mathrm{~Hz}, \mathrm{Ar}-\mathrm{H})$; 7.34-7.39 (m, 2H, Ar-H); 7.49-7.52 (m, 3H, Ar-H); $7.60(\mathrm{~d}, 2 \mathrm{H}, J=8.4 \mathrm{~Hz}, \operatorname{Ar}-\mathrm{H}) ; 7.80(\mathrm{~d}, 2 \mathrm{H}, J=$ $7.6 \mathrm{~Hz}, \mathrm{Ar}-\mathrm{H}) ; 7.91$ (d, $2 \mathrm{H}, J=8.4 \mathrm{~Hz}, \mathrm{Ar}-\mathrm{H}) ; 8.68$ (s, $1 \mathrm{H}$, pyrazolyl-H). ${ }^{13} \mathrm{C}$ NMR $\left(125 \mathrm{MHz}, \mathrm{CDCl}_{3}\right) \delta_{\mathrm{C}}$ 21.6, 109.7, 110.4, 119.8, 123.3, 126.1, 127.7, 129.7, 130.9, 131.1, 131.4. MS (EI): $m / z=430\left[\mathrm{M}^{+}\right], 432$ $\left[\mathrm{M}^{+2}\right]$. Anal. Calcd for $\mathrm{C}_{23} \mathrm{H}_{15} \mathrm{BrN}_{3} \mathrm{O}: \mathrm{C}, 64.20 ; \mathrm{H}$, 3.75 ; N, 9.77\%. Found: C, 64.25; H, 3.69; N, 10.02\%.

2.3y 2-[3-(4-Ethoxyphenyl)-1-phenyl-1H-pyrazole-4yl]benzo[d]oxazole $\left(\mathbf{2} \boldsymbol{y}^{\prime}\right)$ : Orange solid; mp 189$191^{\circ} \mathrm{C} ; R_{f}=0.44$ (AcOEt/petroleumether 50\%). IR (KBr): 3430, 2930, 1631, 1583, 1450, 1240, 1045,
$750 \mathrm{~cm}^{-1} .{ }^{1} \mathrm{H}$ NMR $\left(500 \mathrm{MHz}, \mathrm{CDCl}_{3}\right) \delta_{\mathrm{H}} 1.43(\mathrm{t}, 3 \mathrm{H}$, $\left.J=6.9 \mathrm{~Hz},-\mathrm{OCH}_{2} \mathrm{CH}_{3}\right) ; 4.09(\mathrm{q}, 2 \mathrm{H}, J=6.9 \mathrm{~Hz}$, $\left.-\mathrm{OCH}_{2} \mathrm{CH}_{3}\right) ; 6.98(\mathrm{~d}, 2 \mathrm{H}, J=9.1 \mathrm{~Hz}, \mathrm{Ar}-\mathrm{H}) ; 7.26-$ 7.29 (m, 2H, Ar-H); 7.34 (t, 1H, $J=7.6 \mathrm{~Hz}, \mathrm{Ar}-\mathrm{H})$; 7.46-7.54 (m, 3H, Ar-H); 7.69 (d, $1 \mathrm{H}, J=6.9 \mathrm{~Hz}, \mathrm{Ar}-$ $\mathrm{H}) ; 7.80(\mathrm{~d}, 2 \mathrm{H}, J=7.6 \mathrm{~Hz}, \operatorname{Ar}-\mathrm{H}) ; 7.94(\mathrm{~d}, 2 \mathrm{H}, J=$ $8.4 \mathrm{~Hz}, \mathrm{Ar}-\mathrm{H}) ; 8.68$ (s, 1H, pyrazolyl-H). ${ }^{13} \mathrm{C} \mathrm{NMR}$ $\left(125 \mathrm{MHz}, \mathrm{CDCl}_{3}\right) \delta_{\mathrm{C}} 14.9,63.6,109.9,110.4,114.3$, $119.4,119.8,124.5,124.7,127.4,129.7,130.2$, 130.6, 139.4, 142.0, 147.0, 150.2, 152.1, 158.6, 159.7. MS (EI): $m / z=381\left[\mathrm{M}^{+}\right]$. Anal. Calcd for $\mathrm{C}_{24} \mathrm{H}_{19} \mathrm{~N}_{3} \mathrm{O}_{2}$ : C, 75.57; H, 5.02; N, 11.02\%. Found: C, 75.44; H, $5.11 ; \mathrm{N}, 11.09 \%$.

$2.3 \mathrm{z} \quad$ 5-Chloro-2-phenylethylbenzo[d]oxazole $\left(2 z^{\prime}\right)$ : Yellow solid; mp $114-116^{\circ} \mathrm{C} ; \quad R_{f}=0.50$ (AcOEt/petroleumether 40\%). IR (KBr): 3402, 1621, 1585, 1488, 1391, 1239, 1088, $985 \mathrm{~cm}^{-1}$. ${ }^{1} \mathrm{H}$ NMR $\left(500 \mathrm{MHz}, \mathrm{CDCl}_{3}\right) \delta_{\mathrm{H}} 3.14(\mathrm{t}, 2 \mathrm{H}, J=7.6 \mathrm{~Hz}) ; 3.29(\mathrm{t}$, $2 \mathrm{H}, J=7.6 \mathrm{~Hz}) ; 7.15-7.20(\mathrm{~m}, 1 \mathrm{H}, \mathrm{Ar}-\mathrm{H}) ; 7.24-7.28$ $(\mathrm{m}, 4 \mathrm{H}, \mathrm{Ar}-\mathrm{H}) ; 7.38(\mathrm{dd}, 1 \mathrm{H}, J=2.1,8.6 \mathrm{~Hz}, \mathrm{Ar}-\mathrm{H})$; $7.71(\mathrm{~d}, 1 \mathrm{H}, J=8.6 \mathrm{~Hz}, \operatorname{Ar}-\mathrm{H}) ; 7.75-7.77(\mathrm{~m}, 1 \mathrm{H}$, Ar-H). ${ }^{13} \mathrm{C}$ NMR $\left(125 \mathrm{MHz}, \mathrm{CDCl}_{3}\right) \delta_{\mathrm{C}} 29.4,31.6$, $111.7,119.0,124.6,126.2,128.2,128.3,128.4,140.0$, 142.1, 148.9, 167.9. MS (EI): $m / z=257\left[\mathrm{M}^{+}\right], 259$ $\left[\mathrm{M}^{+2}\right]$. Anal. Calcd for $\mathrm{C}_{24} \mathrm{H}_{19} \mathrm{~N}_{3} \mathrm{O}_{2}: \mathrm{C}, 69.91 ; \mathrm{H}, 4.69$; N, 5.43\%. Found: C, 70.07; H, 4.65; N, 5.47\%.

\subsection{Animals and drug dosage}

2.4a Animals: The selection of animals, caring and handling was done as per the guidelines set by the Indian National Science Academy, New Delhi, India. Inbred albino mice (Swiss strain) of adult gender weighing $120-150 \mathrm{~g}$ were used for the study. The mice were housed individually in clean polypropylene cages containing sterile paddy husk (procured locally) as bedding throughout the experiment. All animals were fed with sterile commercial pelleted rat chow supplied by Hindustan Lever Ltd. (Mumbai, India) with free access to water (ad libitum) under standardized housing conditions (natural light-dark cycle, temperature $23 \pm 1{ }^{\circ} \mathrm{C}$, relative humidity $55 \pm 5 \%$ ). After 7 days of adaptation to laboratory conditions, the animals were randomly assigned to 12 experimental groups of 5 mice each. Each mouse was used only once. All tests were performed between 08:00 and 16:00 h. All efforts were made to minimize animal suffering and to use only the number of animals necessary to produce reliable scientific data. The experimental protocols and procedures listed below conformed to the Guide for the Care and 
Use of Laboratory Animals and approved by the Institutional Ethics Committee. Mice equivalent doses in $\mathrm{mg} / \mathrm{kg}$ body weight of clinical doses were calculated as $\mathrm{mg} / \mathrm{kg}$ body weight with the help of standard tables (Karber's method). ${ }^{59}$

2.4b Dose and administration of compounds: The synthesized compounds $(50 \mathrm{mg} / \mathrm{kg})$, pentazocine as a reference opiod analgesic drug $(50 \mathrm{mg} / \mathrm{kg})$ and $2 \%$ gum acacia as control were administered orally by intragastric tube.

\subsection{Statistical analysis}

The obtained data were analysed using one-way analysis of variance (ANOVA) followed by Dunnet's multiple comparison test using computerized Graph Pad Instat version 3.05 (Graph Pad software, USA). The results are presented as mean \pm Standard error of means (SEM). Differences between data sets were considered as significant when $P<0.001$.

\section{Results and discussion}

\subsection{Chemistry}

It is pertinent to note that in our earlier study, a twostep approach for the synthesis of a series of benzoxazoles and benzothiazoles have been described via PCC promoted oxidative cyclization of phenolic and thiophenolic imines, respectively (scheme 1). ${ }^{58 a}$

Given the significance of this methodology, we were interested in a one-pot process by combining the reactions such as condensation and oxidation with particular emphasis on performing this transformation under microwave condition using an alternate oxidant. The choice of the oxidant plays a crucial role, since we wished a particular oxidant to be capable of promoting the oxidative cyclization of both thiophenolic and phenolic schiff bases. The IBD (iodobenzene diacetate) promoted synthesis of substituted benzoxazoles through oxidative intramolecular cyclization of the

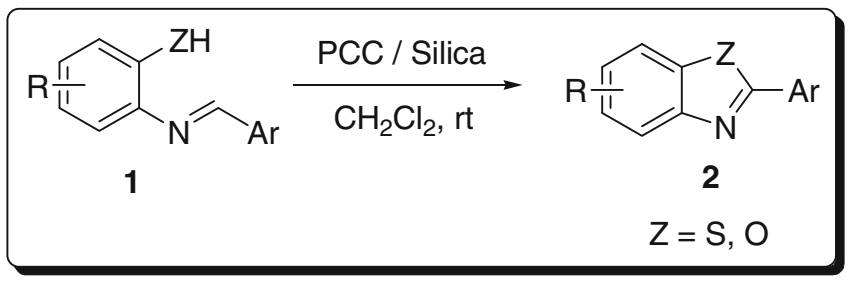

Scheme 1. PCC promoted synthesis of benz(oxa)thiazoles. corresponding phenolic imines was particularly attractive, since this reaction utilizes a mild oxidant. ${ }^{42}$ However, this protocol was amenable to the synthesis of only benzoxazoles and no synthesis of benzothiazoles was reported. It was anticipated that similar oxidant, PIFA [Phenyliodonium bis(trifluoroacetate)] could promote the oxidative cyclization of phenolic and thiophenolic imines. The oxidizing capability of PIFA in organic synthesis is well-documented but recent developments have seen a host of further applications. ${ }^{60}$

To begin our studies, we proceeded to explore the PIFA $(1.05 \mathrm{mmol})$ promoted oxidative cyclization reaction of 2-aminothiophenol $(1.1 \mathrm{mmol})$ with $p$ anisaldehyde $(1.0 \mathrm{mmol})$ in ethanol ${ }^{61}$ at $80^{\circ} \mathrm{C}$ under microwave irradiation. To our delight, the reaction was complete after $5 \mathrm{~min}$ and showed a good conversion towards benzothiazole $\mathbf{2 a}$, which was isolated in $60 \%$ yield after aqueous work-up followed by column chromatography. This positive initial result prompted us to further investigate the conditions suitable for this reaction under microwave irradiation. Extension of the irradiation time from to $15 \mathrm{~min}$ resulted in the complete conversion and $\mathbf{2 a}$ was isolated in $80 \%$ yield. Further irradiation up to $30 \mathrm{~min}$ did not lead to the increase in product yield. Attempts to decrease the reaction temperature were unsuccessful. Starting from these observations, we chose microwave irradiation of the substrates with PIFA $(1.05 \mathrm{mmol})$ in ethanol at $80^{\circ} \mathrm{C}$ for $15 \mathrm{~min}$ as the standard reaction conditions for the synthesis of a wide range of benzothiazoles (scheme 2) (see supporting information). The versatility of this methodology was demonstrated with respect to variation in the aldehyde and amine by synthesis of a small family of benzothiazoles $\mathbf{2 a}-\mathbf{2 z}$ (figure 1). As shown in figure 1, our microwave-assisted oxidative cyclization worked well for a variety of aldehydes and 2-aminothiophenols, giving good to excellent yields of the corresponding benzothiazoles 2a-2z. However, compounds (2r, 2s and 2t) containing heterocyclic cores like pyridine, thiophene and furan, respectively were obtained only in moderate yields. These results can be attributed to the cleavage of these heterocycles under microwave condition. However, other microwave-assisted protocols resulted in excellent yield of similar products. ${ }^{28 e}$

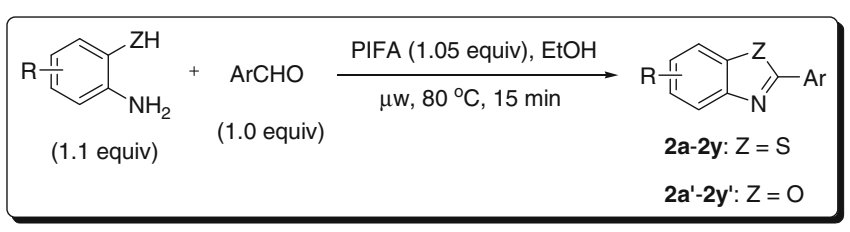

Scheme 2. PIFA promoted synthesis of benz(oxa)thiazoles under microwave irradiation. 


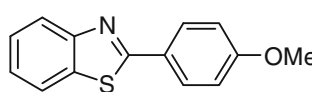

2a $80 \%$

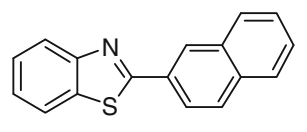

2e $\quad 70 \%$

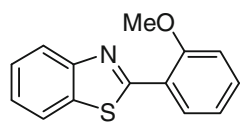

2i $79 \%$<smiles>COc1cc(-c2nc3ccccc3s2)cc(OC)c1O</smiles>

$2 \mathrm{~m} \quad 75 \%$

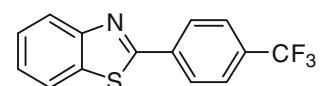

2b $90 \%$

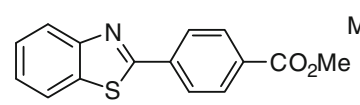

2 f $81 \%$

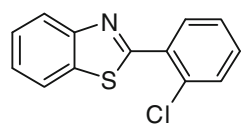

2j $82 \%$

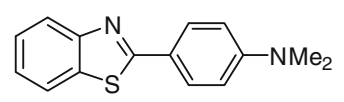

2c $71 \%$

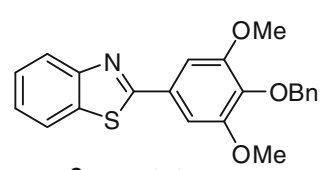

2n $76 \%$

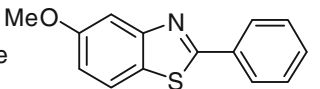

$2 g \quad 92 \%$

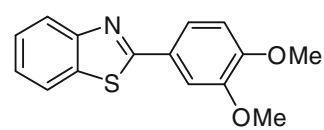

2k $79 \%$

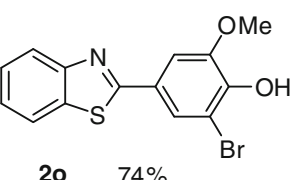

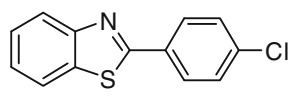

2d $88 \%$
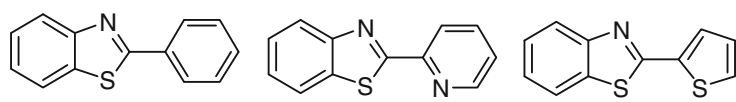

$2 r \quad 63 \%$

2s $61 \%$

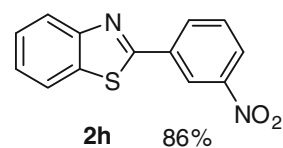

$2 q \quad 87 \%$

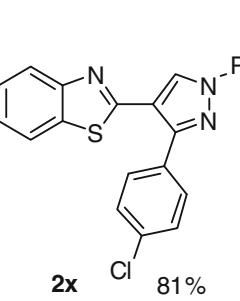

2t $\quad 59 \%$

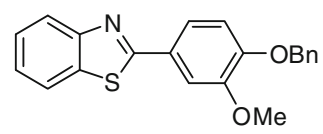

21 $77 \%$
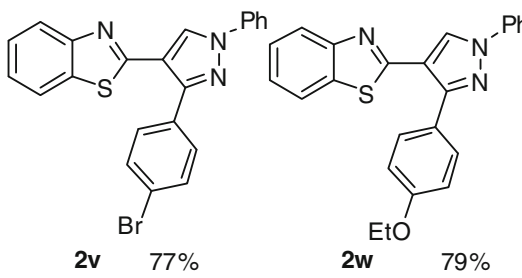

2x
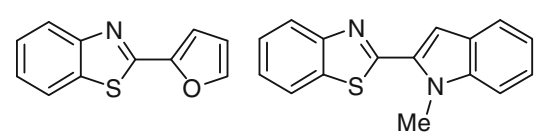

$2 p \quad 84 \%$

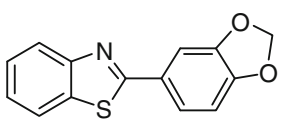

$2 \mathrm{u} \quad 74 \%$

Figure 1. Library of benzothiazoles $\mathbf{2 a}-\mathbf{2 z}$ and their isolated yield (\%).

To study the scope of this reaction, a range of 2aminophenols and aldehydes carrying different functional groups were subjected under the same reaction condition. The results obtained are summarized in figure 2. As shown in figure 2, the substrates possessing both electron releasing and electron donating groups were compatible with this microwave-assisted protocol giving good to excellent yields of the corresponding benzoxazole derivatives $\mathbf{2} \mathbf{a}^{\prime}-\mathbf{2} \mathbf{z}^{\prime}$ (see supporting information). Similar to the benzothiazole series, benzoxazoles possessing furan, thiophene and pyrrolle $\left(\mathbf{2} \mathbf{s}^{\prime}, \mathbf{2} \mathbf{t}^{\prime}\right.$ and $\mathbf{2} \mathbf{u}^{\prime}$ ) were obtained in moderate yields. The structure of all the synthesized compounds was confirmed by spectral data (IR, ${ }^{1} \mathrm{H}$ NMR ${ }^{13} \mathrm{C}$ NMR and EI-MS) and elemental analyses (see supplementary information).

The formation of benzoxa(thia)zole derivatives can be explained by scheme 3 . Reaction of aldehyde with 2-aminothiophenol/2-aminophenol produces the imine intermediate $\mathbf{1}$. The attack of the imino nitrogen of intermediate $\mathbf{1}$ on the Lewis acidic trivalent iodine makes the adjacent carbon more electrophilic, thus it makes way for the facile attack of nucleophilic Z (S or $\mathrm{O}$ ) atom leading to the benzoxa(thia)zoline intermediate 1a. Subsequent dehydrogenation affords the benzoxa(thia)zole product $\mathbf{2}$ along with iodobenzene and trifluoroacetic acid as by-product. From the mechanism it was evident that PIFA serves both as a Lewis acid as well as an oxidant.

\section{Pharmacology}

\subsection{Evaluation of in vivo analgesic activity}

Ten compounds of benzothiazoles $(\mathbf{2 h}, \mathbf{2} \mathbf{j}, \mathbf{2} \mathbf{l}, \mathbf{2 n}, \mathbf{2 0}$ and $\mathbf{2} \mathbf{u}-\mathbf{2 y})$ and benzoxazoles $\left(\mathbf{2} \mathbf{a}^{\prime}, \mathbf{2} \mathbf{c}^{\prime}, \mathbf{2} \mathbf{h}^{\prime}, \mathbf{2} \mathbf{p}^{\prime}, \mathbf{2} \mathbf{r}^{\prime}\right.$ and $\mathbf{2} \mathbf{u}^{\prime}-\mathbf{2} \mathbf{y}^{\prime}$ ), respectively were selected to evaluate the analgesic activity. To begin with the oral toxicity of the synthesized compounds was performed by acute toxic class method. ${ }^{59}$ The selected adult albino rats were used to determine the dose. The animals were 


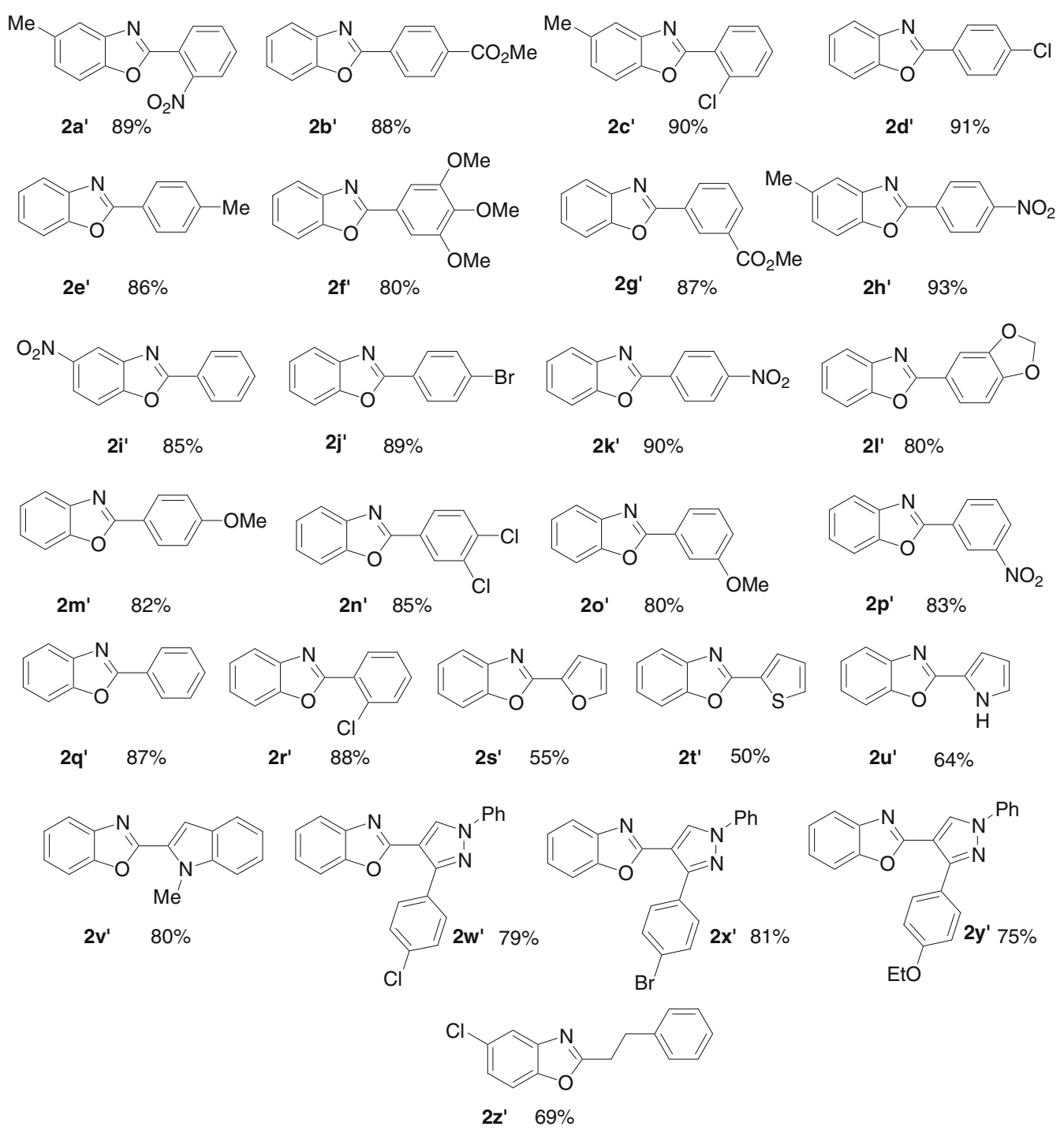

Figure 2. Library of benzoxazoles $\mathbf{2} \mathbf{a}^{\prime}-\mathbf{2} \mathbf{z}^{\prime}$ and their isolated yield (\%).

fasted overnight prior to the acute experimental procedure. Following the period of fasting, the animals were weighed and the synthesized compounds were orally administered at a dose of $50 \mathrm{mg} / \mathrm{kg}$ body weight. Immediately after dosing, the animals were observed continuosly for the first $30 \mathrm{~min}$ for behavioural changes and for mortality at the end of $24 \mathrm{~h}, 48 \mathrm{~h}, 72 \mathrm{~h}$ and $96 \mathrm{~h}$, respec- tively. As no mortality was observed with the above dose even after $96 \mathrm{~h}$, the $\mathrm{LD}_{50}$ value of the compounds expected to exceed $50 \mathrm{mg} / \mathrm{kg}$ body weight. Toxicity assays showed that all the compounds proved to be non-toxic at tested dose levels and well-tolerated by the experimental animals as their $\mathrm{LD}_{50}$ cut-off values $>50 \mathrm{mg} / \mathrm{kg}$ body weight.

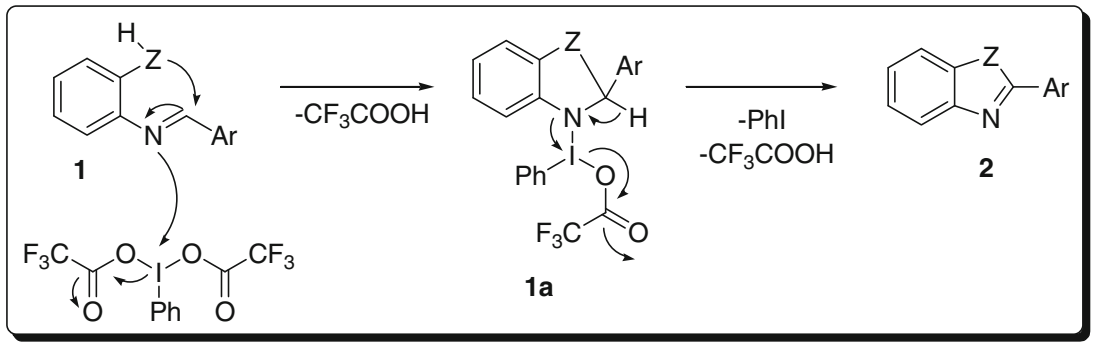

Scheme 3. Plausible mechanism for the formation of benz(oxa)thiazoles 2. 
Table 1. Analgesic activity of selected compounds by tail immersion test.

\begin{tabular}{|c|c|c|c|c|c|c|c|c|}
\hline \multirow[b]{2}{*}{ Entry } & \multirow[b]{2}{*}{ Treatments } & \multirow{2}{*}{$\begin{array}{l}\text { Dose } \\
\text { levels }\end{array}$} & \multicolumn{6}{|c|}{ Tail immersion response in seconds (mean \pm SEM) } \\
\hline & & & $0 \min$ & $15 \min$ & $30 \mathrm{~min}$ & $60 \mathrm{~min}$ & $90 \mathrm{~min}$ & $\%$ potency \\
\hline 1 & $2 \mathbf{h}$ & $50 \mathrm{mg} / \mathrm{kg}$ & $1.31 \pm 0.01$ & $2.46 \pm 0.02$ & $2.52 \pm 0.02$ & $2.56 \pm 0.02$ & $2.61 \pm 0.01 *$ & 50.0 \\
\hline 2 & $2 \mathbf{j}$ & $50 \mathrm{mg} / \mathrm{kg}$ & $1.30 \pm 0.01$ & $2.46 \pm 0.02$ & $2.50 \pm 0.02$ & $2.55 \pm 0.02$ & $2.61 \pm 0.02 *$ & 50.0 \\
\hline 3 & 21 & $50 \mathrm{mg} / \mathrm{kg}$ & $1.30 \pm 0.01$ & $2.50 \pm 0.01$ & $2.54 \pm 0.04$ & $2.60 \pm 0.02$ & $2.67 \pm 0.01 *$ & 51.3 \\
\hline 4 & $2 n$ & $50 \mathrm{mg} / \mathrm{kg}$ & $1.32 \pm 0.01$ & $2.66 \pm 0.02$ & $2.69 \pm 0.02$ & $2.73 \pm 0.03$ & $2.78 \pm 0.03 *$ & 52.5 \\
\hline 5 & 20 & $50 \mathrm{mg} / \mathrm{kg}$ & $1.31 \pm 0.01$ & $2.33 \pm 0.02$ & $2.38 \pm 0.01$ & $2.62 \pm 0.09$ & $2.78 \pm 0.08 *$ & 52.8 \\
\hline 6 & $2 u$ & $50 \mathrm{mg} / \mathrm{kg}$ & $1.30 \pm 0.01$ & $2.63 \pm 0.01$ & $2.66 \pm 0.03$ & $2.83 \pm 0.01$ & $3.09 \pm 0.08 *$ & 58.0 \\
\hline 7 & $2 \mathbf{v}$ & $50 \mathrm{mg} / \mathrm{kg}$ & $1.30 \pm 0.01$ & $2.68 \pm 0.02$ & $3.52 \pm 0.01$ & $4.10 \pm 0.03$ & $4.98 \pm 0.02 *$ & 73.9 \\
\hline 8 & $2 w$ & $50 \mathrm{mg} / \mathrm{kg}$ & $1.31 \pm 0.01$ & $2.69 \pm 0.01$ & $3.62 \pm 0.01$ & $4.37 \pm 0.04$ & $5.00 \pm 0.01 *$ & 73.8 \\
\hline 9 & $2 x$ & $50 \mathrm{mg} / \mathrm{kg}$ & $1.30 \pm 0.01$ & $2.55 \pm 0.02$ & $3.49 \pm 0.01$ & $3.99 \pm 0.03$ & $4.70 \pm 0.02 *$ & 72.3 \\
\hline 10 & $2 y$ & $50 \mathrm{mg} / \mathrm{kg}$ & $1.31 \pm 0.01$ & $2.59 \pm 0.02$ & $3.59 \pm 0.02$ & $4.00 \pm 0.02$ & $4.64 \pm 0.03 *$ & 71.7 \\
\hline 11 & $2 \mathbf{a}^{\prime}$ & $50 \mathrm{mg} / \mathrm{kg}$ & $1.30 \pm 0.01$ & $2.45 \pm 0.02$ & $2.56 \pm 0.03$ & $2.60 \pm 0.02$ & $2.63 \pm 0.01 *$ & 50.6 \\
\hline 12 & $2 c^{\prime}$ & $50 \mathrm{mg} / \mathrm{kg}$ & $1.30 \pm 0.01$ & $2.46 \pm 0.01$ & $2.50 \pm 0.02$ & $2.59 \pm 0.01$ & $2.65 \pm 0.01 *$ & 50.9 \\
\hline 13 & $2 \mathbf{h}^{\prime}$ & $50 \mathrm{mg} / \mathrm{kg}$ & $1.30 \pm 0.01$ & $2.47 \pm 0.01$ & $2.60 \pm 0.01$ & $2.64 \pm 0.01$ & $2.67 \pm 0.01 *$ & 51.3 \\
\hline 14 & $2 \mathbf{p}^{\prime}$ & $50 \mathrm{mg} / \mathrm{kg}$ & $1.31 \pm 0.01$ & $2.49 \pm 0.03$ & $2.59 \pm 0.2$ & $2.64 \pm 0.01$ & $2.69 \pm 0.02 *$ & 51.3 \\
\hline 15 & $2 r^{\prime}$ & $50 \mathrm{mg} / \mathrm{kg}$ & $1.30 \pm 0.01$ & $2.38 \pm 0.02$ & $2.44 \pm 0.01$ & $2.51 \pm 0.01$ & $2.60 \pm 0.01 *$ & 50.0 \\
\hline 16 & $2 u^{\prime}$ & $50 \mathrm{mg} / \mathrm{kg}$ & $1.30 \pm 0.01$ & $2.37 \pm 0.02$ & $2.43 \pm 0.03$ & $2.70 \pm 0.09$ & $2.86 \pm 0.08 *$ & 54.5 \\
\hline 17 & $2 \mathbf{v}^{\prime}$ & $50 \mathrm{mg} / \mathrm{kg}$ & $1.30 \pm 0.01$ & $2.69 \pm 0.01$ & $3.01 \pm 0.01$ & $3.13 \pm 0.01$ & $3.22 \pm 0.08 *$ & 59.6 \\
\hline 18 & $2 \mathbf{w}^{\prime}$ & $50 \mathrm{mg} / \mathrm{kg}$ & $1.32 \pm 0.01$ & $2.60 \pm 0.02$ & $3.37 \pm 0.03$ & $4.12 \pm 0.01$ & $4.99 \pm 0.01 *$ & 73.5 \\
\hline 19 & $2 x^{\prime}$ & $50 \mathrm{mg} / \mathrm{kg}$ & $1.31 \pm 0.01$ & $2.78 \pm 0.01$ & $3.57 \pm 0.03$ & $4.31 \pm 0.01$ & $5.02 \pm 0.01 *$ & 76.4 \\
\hline 20 & $2 \mathbf{y}^{\prime}$ & $50 \mathrm{mg} / \mathrm{kg}$ & $1.30 \pm 0.01$ & $2.67 \pm 0.02$ & $3.45 \pm 0.01$ & $4.25 \pm 0.03$ & $5.00 \pm 0.02 *$ & 74.0 \\
\hline 21 & Gum acacia ${ }^{a}$ & $2 \mathrm{~mL} / \mathrm{kg}$ & $1.30 \pm 0.01$ & $1.24 \pm 0.01$ & $1.12 \pm 0.01$ & $1.15 \pm 0.01$ & $1.30 \pm 0.01 *$ & - \\
\hline 22 & Pentazocine & $50 \mathrm{mg} / \mathrm{kg}$ & $1.30 \pm 0.01$ & $6.31 \pm 0.03$ & $6.39 \pm 0.04$ & $6.54 \pm 0.03$ & $6.72 \pm 0.02 *$ & 80.6 \\
\hline
\end{tabular}

Data were analysed by one way ANOVA followed by Dunnet's test; *indicates $p<0.001$; SEM: Standard error of means. a $2 \%(w / v)$ of gum acacia was used as control.

Analgesic activity of the synthesized compounds was determined using tail immersion method. ${ }^{62}$ Healthy Swiss mice $(n=6)$ of either sex was selected by random sampling technique and placed into individual restraining cages leaving the tail hanging out freely. The animals were then allowed to adapt in the cages for 30 min before testing. The lower $5 \mathrm{~cm}$ portion of the tail was marked and immersed in a beaker of freshly filled warm water of at $55 \pm 5^{\circ} \mathrm{C}$. Within a few seconds the rat reacted by withdrawing the tail. The reaction time was recorded by a stop watch. After each determination the tail was carefully dried. This reaction was determined before oral feeding of the drug and synthesized compounds which were recorded as zero minutes reading. The test compounds, control (2\% gum acacia) and standard (pentazocine) at a dose level of $50 \mathrm{mg} / \mathrm{kg}$ body weight were administered orally by intragastric tube. The time (in seconds) to withdraw the tail clearly out of water was taken as the reaction time. The first reading $(0 \mathrm{~min})$ was taken immediately after the administration of the test compound and subsequent reaction time was recorded at 15, 30, 60 and $90 \mathrm{~min}$, respectively. The cut-off time of the immersion is $15 \mathrm{~s}$. The mean reaction time was recorded for each group and compared with the value of the standard drug pentazocine. The percentage analgesic activity was calculated using the formula:

$$
\% \text { potency }=\left[\left(T_{2}-T_{1}\right) / T_{2}\right] \times 100,
$$

where, $T_{1}$ is the reaction time (in sec) before treatment and $T_{2}$ is the reaction time (in seconds) after treatment.

The results of analgesic activity are presented in table 1, which demonstrate that 2-aryl benz(oxa)thiazole analogues (2h-2o) were generally found to be less potent than their corresponding heteroaryl analogues $\left(\mathbf{2} \mathbf{u}-\mathbf{2 y}\right.$ and $\left.\mathbf{2} \mathbf{u}^{\prime}-\mathbf{2} \mathbf{y}^{\prime}\right)$. Among the heteroaryl analogues, pyrazolyl groups $(\mathbf{2 v}-\mathbf{2 y}$ and $\mathbf{2} \mathbf{w}^{\prime}-\mathbf{2} \mathbf{y}^{\prime}$ ) exhibited good analgesic activity and their values are comparable to the standard pentazocine. Compounds adjoined with indolyl $\left(\mathbf{2} \mathbf{u}\right.$ and $\left.\mathbf{2} \mathbf{v}^{\prime}\right)$ and pyrollyl motifs $\left(\mathbf{2} \mathbf{u}^{\prime}\right)$ showed moderate potency with activities greater than 2-aryl analogues but lesser than 2-pyrazolyl analogues.

\section{Conclusion}

In summary, we have explored a useful and practical approach to benzoxazoles and benzothiazoles by PIFA 
promoted cyclocondensation of 2-aminothiophenol/2aminophenol with aldehydes. The current protocol is noteworthy; since it has advantages like wide substrate scope, short reaction time, microwave condition and satisfactory yields. Evaluation of analgesic activity of twenty compounds was performed by tail immersion test. All the tested compounds displayed varying degrees of analgesic activity. Benz(oxa)thiazole derivatives bearing pyrazolyl system exhibited comparable to or slightly less potent activity than the standard pentazocine.

\section{Supplementary information}

For supplementary information see www.ias.ac.in/ chemsci.

\section{Acknowledgements}

The author CP thanks the Council of Scientific and Industrial Research (CSIR), New Delhi, India, for the research fellowship. PD thanks Dr. K Karthikeyan, Organic Chemistry Division, Central Leather Research Institute, Chennai for recording NMR spectrum.

\section{References}

1. (a) Temiz O, Oren I, Sener E, Yalcin I and Ucarturk N 1998 Farmaco 53 337; (b) Sato S, Kajiura T, Noguchi M, Takehan K, Kobayashi T and Tsuji T 2001 J. Antibiot. 54 102; (c) Bradshaw T D, Shi D F, Schultz R J, Paull K D, Kelland L, Wilson A, Garner C, Fiebig H H, Wrigley S and Stevens M F 1998 Br. J. Cancer 78 421; (d) Craigo W A, LeSueur B W and Skibo E B 1999 J. Med. Chem. 42 3324; (e) Gudmundsson K S, Tidwell J, Lippa N, Koszalka G W, van Draanen N, Ptak R G, Drach J C and Townsend L B 2000 J. Med. Chem. 43 2464; (f) Chen J J, Wei Y, Drach J C and Townsend L B 2000 J. Med. Chem. 43 2449; (g) Mann J, Baron A, Opoku-Boahen Y, Johansson E, Parkinson G, Kelland L R and Neidle S 2001 J. Med. Chem. 44 138; (h) Kuhler T C, Swanson M, Christenson B, Klintenberg A-C, Lamm B, Fagerhag J, Gatti R, Olwegard-Halvarsson M, Shcherbuchin V, Elebring T and Sjostrom J-E $2002 \mathrm{~J}$. Med. Chem. 45 4282; (i) Katsura Y, Inoue Y, Nishino S, Tomoi M, Itoh H and Takasugi H 1992 Chem. Pharm. Bull. 40 1424; (j) Koci J, Klimesova V, Waisser K, Kaustova J, Dahse H M and Mollmann U 2002 Bioorg. Med. Chem. Lett. 12 3275; (k) Katsura Y, Inoue Y, Tomishi T, Itoh H, Ishikawa H and Takasugi H 1992 Chem. Pharm. Bull. 402432

2. Kashiyama E, Hutchinson I, Chua M S, Stinson S F, Phillips L R, Kaur G, Sausville E A, Bradshaw T D, Westwell A D and Stevens M F G 1999 J. Med. Chem. 424172

3. Lau C K, Dufresne C, Gareau Y, Zamboni R, Labelle M, Young R N, Metters K M, Rochette C, Sawyer N, Slipetz
D M, Charette L, Jones T, McAuliffe M, McFarlane C and Ford-Hutchinson A W 1995 Bioorg. Med. Chem. 51615

4. Bergman J M, Coleman P J, Cox C, Hartman G D, Lindsley C, Mercer S P, Roecker A J and Whitman D B 2006 PCT Int. Appl. WO 2006127550

5. Nagel A A, Liston D R, Jung S, Maher M, Vincent L A, Chapin D, Chen Y L, Hubbard S, Ives J L and Jones S B 1995 J. Med. Chem. 381084

6. (a) Chua M-S, Shi D-F, Wrigley S, Bradshaw T D, Hutchinson I, Shaw P N, Barrett D A, Stanley L A and Stevens M F G 1999 J. Med. Chem. 42 381; (b) hutchinson I, Chua M-S, Browne H L, Trapani V, Bradshaw T D, Westwell A D and Stevens M F G 2001 J. Med. Chem. 44 1446; (c) Leng W, Zhou Y, Xu Q and Liu J 2001 Macromolecules 34 4774; (d) Hutchinson I, Jennings S A, Vishnuvajjala B R, Westwell A D and Stevens M F G 2002 J. Med. Chem. 45744

7. (a) Shi D-F, Bradshaw T D, Wrigley S, McCall C J, Lelieveld P, Fichtner I and Stevens M F G 1996 J. Med. Chem. 39 3375; (b) Beebe X, Wodka D and Sowin T J 2001 J. Comb. Chem. 3 360; (c) Hari A, Karan C, Rodrigues W C and Miller B L $2001 \mathrm{~J}$. Org. Chem. 66 991; (d) Pottorf R S, Chadha N K, Katkevics M, Ozola V, Suna E, Ghane H, Regberg T and Player M R 2003 Tetrahedron Lett. 44175

8. Naitoh T, Mishima M, Kawaguchi S, Matsui K, Andoh T, Kagei K, Kakiki M, Yuzuriha T and Horie T 1997 Xenobiotica 271053

9. Kagaya T, Kajiwara A, Nagato S, Akasaka K and Kubota A 1996 J. Pharmacol. Exp. Ther. 278243

10. Hadjipavlou-Litina D J and Geronikaki A A 1997 Drug Des. Discov. 15199

11. Gong B, Hong F, Kohm C, Bonham L and Klein P 2004 Bioorg. Med. Chem. Lett. 141455

12. (a) Mylari B L, Larson E R, Beyer T A and Zembrowski W J, Aldinger C E, Dee M F, Siegel T W and Singleton D H 1991 J. Med. Chem. 34 108; (b) Kotani T, Nagaki Y, Ishii A, Konishi Y, Yago H, Suehiro S, Okukado N and Okamoto K 1997 J. Med. Chem. 40684

13. Paramashivappa R, Kumar P P, Rao P V S and Rao A S 2003 Bioorg. Med. Chem. Lett. 13657

14. Surgue M F, Gautheron P, Schmitt C, Viader M P, Conquet P, Smith R L, Share N N and Stone C A 1985 J. Pharmacol. Exp. Ther. 232534

15. Sohn S K, Chang M S, Choi W S, Kim K B, Woo T W, Lee S B and Chung Y K 1999 Can. J. Physiol. Pharmacol. 77330

16. Phoon $\mathrm{C}$ W, Ng P Y, Ting A E, Yeo S L and Sim M M 2001 Bioorg. Med. Chem. Lett. 111647

17. Caccese R G, DiJoseph J F, Scotnicki J S, Borella L E and Adams L M 1991 Agents Actions 34223

18. Black C, Deschenes D, Gagnon M, Lachance N, Legar S, C Li S and Oballa R M 2006 PCT Int. Appl. WO 2006122200 A1 20061116

19. (a) Hall I H, Peaty N J, Henry J R, Easmon J, Heinisch G and Purstinger G 1999 Arch. Pharm. (Weinheim) 332 115; (b) Bénéteau V, Besson T, Guillard J, Léonce S and Pfeiffer B 1999 Eur. J. Med. Chem. 34 1053; (c) Hutchinson I, Bradshaw T D, Stevens M F G and Westwell A D 2003 Bioorg. Med. Chem. Lett. 13471

20. (a) Palmer P J, Trigg R B and Warrington J V 1971 J. Med. Chem. 14 248; (b) Ali A, Taylor G E and Graham D W 2001 PCT Int. Appl. WO 2001028561 
21. (a) Ivanov S K and Yuritsyn V S 1971 Neftekhimiya 11 99; (b) Ivanov S K and Yuritsyn V S 1971 Chem. Abstr. $74124487 \mathrm{~m}$

22. (a) Benazzouz A, Boraud T, Dubédat P, Boireau A, Stutzmann J-M and Gross C 1995 Eur. J. Pharmacol. 284 299; (b) Jimonet P, Audiau F, Barreau M, Blanchard J-C, Boireau A, Bour Y, Coléno M-A, Doble A, Doerflinger G, Huu C D, Donat M-H, Duchesne J M, Ganil P, Guérémy C, Honoré E, Just B, Kerphirique R, Gontier S, Hubert P, Laduron P M, Blevec J L, Meunier M, Miquet J-M, Nemecek C, Pasquet M, Piot O, Pratt J, Rataud J, Reibaud M, Stutzmann J-M and Mignani S 1999 J. Med. Chem. 422828

23. (a) McKee M L and Kerwin S M 2008 Bioorg. Med. Chem. 16 1775; (b) Oksuzoglu E, Tekiner-Gulbas B, Alper S, Temiz-Arpaci O, Ertan T, Yildiz I, Diril N, Sener-Aki E and Yalcin I 2008 J. Enzyme Inhib. Med. Chem. 23 37; (c) Oksuzoglu E, Temiz-Arpaci O, Tekiner-Gulbas B, Eroglu H, Sen G, Alper S, Yildiz I, Diril N, Aki-Sener E and Yalcin I 2007 Med. Chem. Res. 16 1; (d) H Potashman M, Bready J, Coxon A, DeMelfi T M, DiPietro Jr L, Doerr N, Elbaum D, Estrada J, Gallant P, Germain J, Gu Y, Harmange J-C, Kaufman S A, Kendall R, Kim J L, Kumar G N, Long A M, Neervannan S, Patel V F, Polverino A, Rose P, Van der Plas S, Whittington D, Zanon R and Zhao H 2007 J. Med. Chem. 50 4351; (e) Huang S-T, Hsei I-J and Chen C 2006 Bioorg. Med. Chem. 146106

24. Deluca M R and Kerwin S M 1997 Tetrahedron Lett. 38 199

25. Edwards P D, Meyer E F, Vijayalakshmi J, Tuthill P A, Andisik D A, Gomes B and Strimpler A 1992 J. Am. Chem. Soc. 1141854

26. Sato Y, Yamada M, Yoshida S, Soneda T, Ishikawa M, Nizato T, Suzuki K and Konno F 1998 J. Med. Chem. 41 3015

27. Rodriguez A D, Ramrez C, Rodiguez I I and Gonzalez E 1999 Org. Lett. 1527

28. (a) Ben-Alloum A, Bakkas S and Soufiaoui M 1997 Tetrahedron Lett. 38 6395; (b) Ranu B C, Jana R and Dey S S 2004 Chem. Lett. 33 274; (c) Mourtas S, Gatos D and Barlos K 2001 Tetrahedron Lett. 42 2201; (d) Itoh T, Nagata K, Ishikawa H and Ohsewa A 2004 Heterocycles 63 2769; (e) Niralwad K S, Shingate B B and Shingare M S 2010 Bull. Korean Chem. Soc. 31 981; (f) Al-Qalaf F, Mekheimer R A and Sadek K U 2008 Molecules 13 2908; (g) Londhe B S, Pratap U R, Mali J R and Mane R A 2010 Bull. Korean Chem. Soc. 31 2329

29. Chen C and Chen Y-J 2004 Tetrahedron Lett. 45113

30. (a) Hutchinson I, Stevens M F G and Westwell A D 2000 Tetrahedron Lett. 41 425; (b) Spitulnik M J 1976 Synthesis 730; (c) Mu X-J, Zou J-P, Zeng R-S and Wu J-C 2005 Tetrahedron Lett. 464345

31. Bose D S and Idrees M 2006 J. Org. Chem. 718261

32. Paul S, Gupta M and Gupta R 2002 Synth. Commun. 32 3541

33. Shirinian V Z, Melkova S Yu, Belenkii L I, Krayushkin M M and Zelinsky N D 2000 Russ. Chem. Bull. 491859

34. Zhong W H, Zhang Y M and Chen X Y 2001 J. Indian Chem. Soc. 78316

35. Roe A and Tucker W P 1965 J. Heterocycl. Chem. 2148
36. Stanetty P and Krumpark B 1996 J. Org. Chem. 615130

37. (a) Ares J J 1991 Synth. Commun. 21 625; (b) Majo V J, Prabhakaran J, Mann J J and Kumar J S D 2003 Tetrahedron Lett. 448535

38. Tale R H 2002 Org. Lett. 41641

39. Hein D W, Alheim R J and Leavitt J J 1957 J. Am. Chem. Soc. 79427

40. Itoh T and Mase T 2007 Org. Lett. 93687

41. Terashima M and Ishii M 1982 Synthesis 1484 and references cited therein

42. (a) Varma R S, Saini R K and Prakash O 1997 Tetrahedron Lett. 38 2621; (b) Prakash O, Pannu K and Kumar A 2006 Molecules 1143

43. Varma R S and Kumar D 1998 J. Heterocycl. Chem. 35 1539

44. Park K H, Jun K, Shin S R and Oh S W 1996 Tetrahedron Lett. 378869

45. Srivastava R G and Venkataramani P S 1988 Synth. Commun. 181537

46. Nakagawa K, Onoue H and Sugita J 1964 Chem. Pharm. Bull. 121135

47. Stephens F F and Bower J D 1949 J. Chem. Soc. 2971

48. Chang J, Zhao K and Pan S 2002 Tetrahedron Lett. 43 951

49. Talapatra S K, Chaudhuri P and Talapatra B 1980 Heterocycles 141279

50. Kidwai M, Bansal V, Saxena A, Aerry S and Mozumdar S 2006 Tetrahedron Lett. 478049

51. Kawashita Y, Nakamichi N, Kawabata H and Hayashi M 2003 Org. Lett. 53713

52. Speier G 1987 J. Mol. Catal. 41253

53. Haginiwa J, Higuchi Y and Ohtsuka H 1976 Yakugaku Zasshi 96209

54. (a) Huxley A 2006 Synlett 2658; (b) Pottorf R S, Chadha N K, Katkevics M, Ozola V, Suna E, Ghane H, Regberg T and Player M R 2003 Tetrahedron Lett. 44175

55. Bonnamour J and Bolm C 2008 Org. Lett. 102665

56. (a) Chen Y and Xie N 2006 J. Photochem. Photobiol. A; Chem. 179 320; (b) Chen Y and Zeng De X 2004 J. Org. Chem. 69 5037; (c) Li Z-X, Fu W-F, Yu M-M, Zhao X-J and Chen Y 2007 Dyes Pigments 75 516; (d) Tauer E and Grellmann K H 1981 J. Org. Chem. 46 4252; (e) Grellmann K H and Tauer E 1967 Tetrahedron Lett. 81909

57. (a) Chen W H and Pang Y 2009 Tetrahedron Lett. 50 6680; (b) Gottumukkala A L, Derridj F, Djebbar S and Doucet H 2008 Tetrahedron Lett. 49 2926; (c) Alagille D, Baldwin R M and Tamagnan G D 2005 Tetrahedron Lett. 46 1349; (d) Nandurkar N S, Bhanushali M J, Bhor M D and Bhanage B M 2008 Tetrahedron Lett. 49 1045; (e) Chen W, Wang M, Li P and Wang L 2011 Tetrahedron 675913

58. (a) Praveen C, Kumar K H, Muralidharan D and Perumal P T 2008 Tetrahedron 64 2369; (b) Praveen C, Kiruthiga P and Perumal P T 2009 Synlett 1990; (c) Praveen C, Karthikeyan K and Perumal P T 2009 Tetrahedron 65 9244; (d) Praveen C, Jegatheesan S and Perumal P T 2009 Synlett 2795; (e) Praveen C, Kalyanasundaram A, and Perumal P T 2010 Synlett 777; (f) Praveen C, Sagayaraj Y W and Perumal P T 2009 Tetrahedron Lett. 50 644; (g) Praveen C, Parthasarathy K and Perumal P T 2010 Synlett 1635; (h) Praveen C, Iyyappan C and 
Perumal P T 2010 Tetrahedron Lett. 51 4767; (i) Praveen C, Dheenkumar P and Perumal P T 2010 Bioorg. Med. Chem. Lett. 20 7292; (j) Praveen C and Perumal P T 2011 Synlett 521; (k) Praveen C, Ayyanar A and Perumal P T 2011 Bioorg. Med. Chem. Lett. 21 4072; (1) Praveen C, Ayyanar A and Perumal P T 2011 Bioorg. Med. Chem. Lett. 21 4170; (m) Praveen C, Iyyappan C, Kumar K S K, Girija K and Perumal P T 2012 J. Chem. Sci. 124451

59. (a) Ghosh M N 2005 Fundamentals of Experimental Pharmacology, 3rd Edition, Hilton and Co., Kolkatta, 190-7; (b) Kale S R and Kale R R 1994 Practical pharmacology and toxicology, 1st Edition, Nirali Prakashan, Pune, 56-57

60. (a) Tohma H, Iwata M and Maegawa T, Kita Y 2002 Tetrahedron Lett. 43 9241; (b) Boldron C, Aromi G,
Challa G, Gamez P and Reedijk J 2005 Chem. Commun. 5808; (c) Quideau S, Lebon M and Lamidey A-M 2002 Org. Lett. 4 3975; (d) Fujioka H, Matsuda S, Horai M, Fujii E, Morishita M, Nishiguchi N, Hata K and Kita Y 2007 Chem. Eur. J. 13 5238; (e) Correa A, Tellitu I, Dominguez E, and SanMartin R 2006 J. Org. Chem. 71 8316; (f) Correa A, Tellitu I, Dominguez E and SanMartin R 2006 Tetrahedron 6211100

61. Since, ethanol has a $\tan \delta$ value of 0.941 ; it was preferable over most of the polar solvents in microwave promoted reactions. For related discussions, see: (a) Galema S A 1997 Chem. Soc. Rev. 26 233; (b) Rajak H and Mishra P 2004 J. Sci. Ind. Res. 63641

62. Vogel H G 2002 Drug discovery and evaluationPharmacological assays, 2nd Edition (New York: Springer) 697 